\title{
APPROXIMATION OF NULL CONTROLS FOR SEMILINEAR HEAT EQUATIONS USING A LEAST-SQUARES APPROACH
}

\author{
Jérôme Lemoine ${ }^{1}$, Irene Marín-Gayte ${ }^{2}$ and Arnaud MÜnCH ${ }^{3, *}$
}

\begin{abstract}
The null distributed controllability of the semilinear heat equation $\partial_{t} y-\Delta y+g(y)=f 1_{\omega}$ assuming that $g \in C^{1}(\mathbb{R})$ satisfies the growth condition $\limsup _{|r| \rightarrow \infty} g(r) /\left(|r| \ln ^{3 / 2}|r|\right)=0$ has been obtained by Fernández-Cara and Zuazua (2000). The proof based on a non constructive fixed point theorem makes use of precise estimates of the observability constant for a linearized heat equation. Assuming that $g^{\prime}$ is bounded and uniformly Hölder continuous on $\mathbb{R}$ with exponent $p \in(0,1]$, we design a constructive proof yielding an explicit sequence converging strongly to a controlled solution for the semilinear equation, at least with order $1+p$ after a finite number of iterations. The method is based on a least-squares approach and coincides with a globally convergent damped Newton method: it guarantees the convergence whatever be the initial element of the sequence. Numerical experiments in the one dimensional setting illustrate our analysis.
\end{abstract}

Mathematics Subject Classification. 35K58, 93B05, 93E24.

Received August 31, 2020. Accepted May 3, 2021.

Paper dedicated to Enrique Zuazua on the occasion of his 60th birthday.

\section{INTRODUCTION}

Let $\Omega \subset \mathbb{R}^{d}, 1 \leq d \leq 3$, be a bounded connected open set whose boundary $\partial \Omega$ is Lipschitz and let $\omega \subset \subset \Omega$ be any non-empty open set. Let $T>0$ and $Q_{T}:=\Omega \times(0, T), q_{T}:=\omega \times(0, T)$ and $\Sigma_{T}:=\partial \Omega \times(0, T)$. We consider the semilinear heat equation

$$
\left\{\begin{array}{l}
\partial_{t} y-\Delta y+g(y)=f 1_{\omega} \quad \text { in } \quad Q_{T}, \\
y=0 \text { on } \Sigma_{T}, \quad y(\cdot, 0)=u_{0} \quad \text { in } \Omega
\end{array}\right.
$$

Keywords and phrases: Semilinear heat equation, null controllability, least-squares method.

${ }^{1}$ Université Clermont Auvergne, Laboratoire de Mathématiques Blaise Pascal CNRS-UMR 6620, Campus des Cézeaux, 63178 Aubière cedex, France.

2 Departamento EDAN, Universidad de Sevilla, Campus Reina Mercedes, 41012 Sevilla, Spain.

3 Université Clermont Auvergne, Laboratoire de Mathématiques Blaise Pascal CNRS-UMR 6620, Campus des Cézeaux, 63178

Aubière cedex, France.

* Corresponding author: arnaud.munch@uca.fr 
where $u_{0} \in L^{2}(\Omega)$ is the initial state of $y$ and $f \in L^{2}\left(q_{T}\right)$ is a control function. Here and throughout the paper, we assume that $g: \mathbb{R} \mapsto \mathbb{R}$ is, at least, locally Lipschitz-continuous and following [16] that $g$ satisfies

$$
\left|g^{\prime}(r)\right| \leq C\left(1+|r|^{m}\right) \quad \text { a.e. with } 1 \leq m \leq 1+4 / d
$$

which implies that (1.1) possesses exactly one local in time solution. We recall that under the additional growth condition

$$
|g(r)| \leq C(1+|r| \log (1+|r|)) \quad \forall r \in \mathbb{R}
$$

(1.1) has a unique globally weak solution defined in $[0, T]$ and one has

$$
y \in C^{0}\left([0, T] ; L^{2}(\Omega)\right) \cap L^{2}\left(0, T ; H_{0}^{1}(\Omega)\right),
$$

see [6]. Without a growth condition of the kind (1.3), the solutions to (1.1) can blow up before $t=T$; in general, the blow-up time depends on $g$ and the size of $\left\|u_{0}\right\|_{L^{2}(\Omega)}$.

The system (1.1) is said to be controllable at time $T$ if, for any $u_{0} \in L^{2}(\Omega)$ and any globally defined bounded trajectory $y^{\star} \in C^{0}\left([0, T] ; L^{2}(\Omega)\right)$ (corresponding to the data $u_{0}^{\star} \in L^{2}(\Omega)$ and $f^{\star} \in L^{2}\left(q_{T}\right)$ ), there exist controls $f \in L^{2}\left(q_{T}\right)$ and associated states $y$ that are again globally defined in $[0, T]$ and satisfy $(1.4)$ and

$$
y(x, T)=y^{\star}(x, T), \quad x \in \Omega .
$$

We refer to [8] for an overview of control problems in nonlinear situations. The uniform controllability strongly depends on the nonlinearity $g$. Fernández-Cara and Zuazua proved in [16] that if $g$ is too "super-linear" at infinity, then, for some initial data, the control cannot compensate the blow-up phenomenon occurring in $\Omega \backslash \bar{\omega}$.

Theorem 1.1 ([16]). There exist locally Lipschitz-continuous functions $g$ with $g(0)=0$ and

$$
|g(r)| \sim|r| \log ^{p}(1+|r|) \quad \text { as } \quad|r| \rightarrow \infty, \quad p>2,
$$

such that (1.1) fails to be controllable for all $T>0$.

On the other hand, Fernández-Cara and Zuazua also proved that if $p$ is small enough, then the controllability holds true uniformly.

Theorem $1.2([16])$. Let $T>0$ be given. Assume that (1.1) admits at least one solution $y^{\star}$, globally defined in $[0, T]$ and bounded in $Q_{T}$. Assume that $g: \mathbb{R} \mapsto \mathbb{R}$ is locally Lipschitz-continuous and satisfies (1.2). If $g$ satisfies

$$
\frac{g(r)}{|r| \ln ^{3 / 2}(1+|r|)} \rightarrow 0 \quad \text { as } \quad|r| \rightarrow \infty
$$

Then (1.1) is controllable at time T.

Therefore, if $|g(r)|$ does not grow at infinity faster than $|r| \ln ^{p}(1+|r|)$ for any $p<3 / 2$, then (1.1) is controllable. We also mention [1] which gives the same result assuming additional sign condition on $g$, namely $g(r) r \geq-C\left(1+r^{2}\right)$ for every $r \in \mathbb{R}$ and some $C>0$. The problem remains open when $g$ behaves at infinity like $|r| \ln ^{p}(1+|r|)$ with $3 / 2 \leq p \leq 2$. We mention however the recent work of Le Balc'h [20] where uniform controllability results are obtained for $p \leq 2$ assuming additional sign conditions on $g$, notably that $g(r)>0$ for $r>0$ or $g(r)<0$ for $r<0$ and $T$ large enough. This condition is not satisfied for $g(r)=-r \ln ^{p}(1+|r|)$. Let us also mention [9] in the context of Theorem 1.1 where a positive boundary controllability result is proved for a specific class of initial and final data and $T$ large enough. 
In the sequel, for simplicity, we shall assume that $g(0)=0, f^{\star} \equiv 0, u_{0}^{\star} \equiv 0$ so that $y^{\star}$ is the null trajectory. The proof given in [16] is based on a fixed point argument that reduced the controllability problem to the obtention of a suitable a priori estimate for the linearized heat equation with a potential. More precisely, it is shown that the operator $\Lambda: L^{\infty}\left(Q_{T}\right) \rightarrow L^{\infty}\left(Q_{T}\right)$, where $y_{z}:=\Lambda(z)$ is a null controlled solution through the control function $f_{z}$ of the linear boundary value problem

$$
\left\{\begin{array}{l}
\partial_{t} y_{z}-\Delta y_{z}+y_{z} \widetilde{g}(z)=f_{z} 1_{\omega} \quad \text { in } \quad Q_{T} \\
y_{z}=0 \text { on } \quad \Sigma_{T}, \quad y_{z}(\cdot, 0)=u_{0} \quad \text { in } \quad \Omega
\end{array}, \quad \widetilde{g}(r):= \begin{cases}\frac{g(r)}{r} & r \neq 0 \\
g^{\prime}(0) & r=0\end{cases}\right.
$$

maps a closed ball $B(0, M) \subset L^{\infty}\left(Q_{T}\right)$ into itself, for some $M>0$. Under the condition (1.6), the Kakutani's theorem provides the existence of at least one fixed point for the operator $\Lambda$, which is also a controlled solution for (1.1).

The main goal of this work is to determine an approximation of the controllability problem associated to (1.1), that is to construct an explicit sequence $\left(f_{k}\right)_{k \in \mathbb{N}}$ converging strongly towards a null control for (1.1). A natural strategy is to take advantage of the method used in $[16,20]$ and consider, for any element $y_{0} \in L^{\infty}\left(Q_{T}\right)$, the Picard iterations $\left(y_{k}\right)_{k \in \mathbb{N}}$ defined by $y_{k+1}:=\Lambda\left(y_{k}\right), k \geq 0$ associated with the operator $\Lambda$. The resulting sequence of controls $\left(f_{k}\right)_{k \in \mathbb{N}}$ is then so that $f_{k+1} \in L^{2}\left(q_{T}\right)$ is a null control for $y_{k+1}$ solution of

$$
\left\{\begin{array}{lll}
\partial_{t} y_{k}-\Delta y_{k}+y_{k} \widetilde{g}\left(y_{k-1}\right)=f_{k} 1_{\omega} & \text { in } & Q_{T}, \\
y_{k}=0 \quad \text { on } \quad \Sigma_{T}, \quad y_{k}(\cdot, 0)=u_{0} & \text { in } \quad \Omega .
\end{array}\right.
$$

Numerical experiments for the one dimensional case reported in [13] exhibit the non convergence of the sequences $\left(y_{k}\right)_{k \in \mathbb{N}}$ and $\left(f_{k}\right)_{k \in \mathbb{N}}$ for some initial conditions large enough. This phenomenon is related to the fact that the operator $\Lambda$ is in general not contracting, even if $\widetilde{g}$ is globally Lipschitz (we refer to Rem. 3.17). We also mention [3, 4] where this strategy is implemented. Still in the one dimensional case, a least-squares type approach, based on the minimization over $L^{2}\left(Q_{T}\right)$ of the functional $\mathcal{R}: L^{2}\left(Q_{T}\right) \rightarrow \mathbb{R}^{+}$defined by $\mathcal{R}(z):=$ $\|z-\Lambda(z)\|_{L^{2}\left(Q_{T}\right)}^{2}$ has been introduced and analyzed in [13]. Assuming that $\widetilde{g} \in C^{1}(\mathbb{R})$ and $g^{\prime} \in L^{\infty}(\mathbb{R})$, it is proved that $\mathcal{R} \in C^{1}\left(L^{2}\left(Q_{T}\right) ; \mathbb{R}^{+}\right)$and that, for some constant $C>0$

$$
\left\|\mathcal{R}^{\prime}(z)\right\|_{L^{2}\left(Q_{T}\right)} \geq\left(1-C\left\|g^{\prime}\right\|_{\infty}\left\|u_{0}\right\|_{\infty}\right) \sqrt{2 \mathcal{R}(z)}, \quad \forall z \in L^{2}\left(Q_{T}\right)
$$

implying that if $\left\|g^{\prime}\right\|_{\infty}\left\|u_{0}\right\|_{\infty}$ is small enough, then any critical point for $\mathcal{R}$ is a fixed point for $\Lambda$ (see [13], Prop. 3.2). Under this smallness assumption on the data, numerical experiments reported in [13] display the convergence of gradient based minimizing sequences for $\mathcal{R}$ and a better behavior than the Picard iterates associated to $\Lambda$. The analysis of convergence is however not performed. As is usual for nonlinear problems and also considered in [13], we may employ a Newton type method to find a zero of the mapping $\widetilde{F}: Y \mapsto W$ defined by

$$
\widetilde{F}(y, f)=\left(\partial_{t} y-\Delta y+g(y)-f 1_{\omega}, y(\cdot, 0)-u_{0}\right) \quad \forall(y, f) \in Y
$$

where the Hilbert space $Y$ and $W$ are defined as follows

$$
Y:=\left\{(y, f): \rho y \in L^{2}\left(Q_{T}\right), \rho_{1} \nabla y \in L^{2}\left(Q_{T}\right)^{d}, \rho_{0}\left(\partial_{t} y-\Delta y\right) \in L^{2}\left(Q_{T}\right), y=0 \text { on } \Sigma_{T}, \rho_{0} f \in L^{2}\left(q_{T}\right)\right\}
$$

and $W:=L^{2}\left(\rho_{0} ; Q_{T}\right) \times L^{2}(\Omega)$ for some appropriates weights $\rho, \rho_{0}$ and $\rho_{1}$. Here $L^{2}\left(\rho_{0} ; Q_{T}\right)$ stands for $\left\{z: \rho_{0} z \in\right.$ $\left.L^{2}\left(Q_{T}\right)\right\}$. It is shown for $d=1$ in [13] that, if $g \in C^{1}(\mathbb{R})$ and $g^{\prime} \in L^{\infty}(\mathbb{R})$, then $\widetilde{F} \in C^{1}(Y ; W)$ allowing to derive 
the Newton iterative sequence: given $\left(y_{0}, f_{0}\right)$ in $Y$, define the sequence $\left(y_{k}, f_{k}\right)_{k \in \mathbb{N}}$ in $Y^{\mathbb{N}}$ iteratively as follows $\left(y_{k+1}, f_{k+1}\right)=\left(y_{k}, f_{k}\right)-\left(Y_{k}, F_{k}\right)$ where $F_{k}$ is a control for $Y_{k}$ solution of

$$
\left\{\begin{array}{l}
\partial_{t} Y_{k}-\Delta Y_{k}+g^{\prime}\left(y_{k}\right) Y_{k}=F_{k} 1_{\omega}+\partial_{t} y_{k}-\Delta y_{k}+g\left(y_{k}\right)-f_{k} 1_{\omega} \quad \text { in } \quad Q_{T} \\
Y_{k}=0 \quad \text { on } \quad \Sigma_{T}, \quad Y_{k}(\cdot, 0)=u_{0}-y_{k}(\cdot, 0) \quad \text { in } \quad \Omega
\end{array}\right.
$$

Once again, numerical experiments for $d=1$ in [13] exhibits the lack of convergence of the Newton method for large enough initial condition, for which the solution $y$ is not close enough to the zero trajectory.

The controllability of nonlinear partial differential equations has attracted a large number of works in the last decades (see the monography [8] and references therein). However, as far as we know, few are concerned with the approximation of exact controls for nonlinear partial differential equations, and the construction of convergent control approximations for controllable nonlinear equations remains a challenge.

Assuming that $g^{\prime} \in L^{\infty}(\mathbb{R})$ and in addition that there exists one $p$ in $(0,1]$ for which $g^{\prime}$ is uniformly Hölder continuous on $\mathbb{R}$ with exponent $p \in(0,1]$, we construct, for any initial data $u_{0} \in L^{2}(\Omega)$, a strongly convergent sequence $\left(f_{k}\right)_{k \in \mathbb{N}}$ towards a control for (1.1). Moreover, after a finite number of iterates related to the norm $\left\|g^{\prime}\right\|_{L^{\infty}(\mathbb{R})}$, the convergence is super linear with a rate equal to $1+p$. This is done (following and improving [26] devoted to a linear case) by introducing a quadratic functional which measures how a pair $(y, f) \in Y$ is close to a controlled solution for (1.1) and then by determining a particular minimizing sequence enjoying the announced property. A natural example of so-called error (or least-squares) functional is given by $\widetilde{E}(y, f):=\frac{1}{2}\|\widetilde{F}(y, f)\|_{W}^{2}$ to be minimized over $Y$. In view of controllability results for (1.1), the non-negative functional $\widetilde{E}$ achieves its global minimum equal to zero for any control pair $(y, f) \in Y$ of $(1.1)$.

The paper is organized as follows. In Section 2, we derive a controllability result for a linearized heat equation with potential in $L^{\infty}\left(Q_{T}\right)$ and source term in $L^{2}\left(0, T ; H^{-1}(\Omega)\right)$. Then, in Section 3, we define the least-squares functional $E$ and the corresponding (nonconvex) optimization problem (3.2) over the Hilbert space $\mathcal{A}$ defined in (3.1). We show that $E$ is Gateaux-differentiable over $\mathcal{A}$ and that any critical point $(y, f)$ for $E$ for which $g^{\prime}(y)$ belongs to $L^{\infty}\left(Q_{T}\right)$ is also a zero of $E$ (see Prop. 3.4). This is done by introducing a descent direction $\left(Y^{1}, F^{1}\right)$ for $E(y, f)$ for which $E^{\prime}(y, f) \cdot\left(Y^{1}, F^{1}\right)$ is proportional to $\sqrt{E(y, f)}$. Then, assuming that the function $g^{\prime}$ is uniformly Hölder continuous on $\mathbb{R}$ with exponent $p$ for some $p \in[0,1]$, we determine a minimizing sequence based on $\left(Y^{1}, F^{1}\right)$ converging strongly to a controlled pair for the semilinear heat equation (1.1). Moreover, we prove that after a finite number of iterates, the convergence enjoys a rate equal to $1+p$ (see Thm. 3.10 for $p=1$ and Thm. 3.12 for $p \in(0,1)$ ). We also emphasize that this least-squares approach coincides with the damped Newton method one may use to find a zero of a mapping similar to $\widetilde{F}$ mentioned above; we refer to Remark 3.15. This explains the convergence of our approach with a super-linear rate. Section 4 gives some numerical illustrations of our result in the one dimensional case and a nonlinear function $g$ uniformly continuous on $\mathbb{R}$ with exponent $p=1$. We conclude in Section 5 with some perspectives.

As far as we know, the analysis of convergence presented in this work, though some restrictive hypotheses on the nonlinear function $g$, is the first one in the context of controllability for partial differential equations.

Along the text, we shall denote by $\|\cdot\|_{\infty}$ the usual norm in $L^{\infty}(\mathbb{R}),(\cdot, \cdot)_{X}$ the scalar product of $X$ (if $X$ is a Hilbert space) and by $\langle\cdot, \cdot\rangle_{X, Y}$ the duality product between the spaces $X$ and $Y$.

\section{A Controllability Result for a linearized heat EQUATion With $L^{2}\left(H^{-1}\right)$ RIGHT HAND SIDE}

We give in this section a controllability result for a linear heat equation with potential in $L^{\infty}\left(Q_{T}\right)$ and right hand side in $L^{2}\left(0, T ; H^{-1}(\Omega)\right)$. As this work concerns the null controllability of parabolic equations, we shall make use of Carleman type weights introduced in this context notably in [17] (we also refer to [12] for a review). 
Here, we assume that such weights $\rho, \rho_{0}, \rho_{1}$ and $\rho_{2}$ blow up as $t \rightarrow T^{-}$and satisfy:

$$
\left\{\begin{array}{l}
\rho=\rho(x, t), \rho_{0}=\rho_{0}(x, t), \rho_{1}=\rho_{1}(x, t) \text { and } \rho_{2}=\rho_{2}(x, t) \text { are continuous and } \geq \rho_{*}>0 \text { in } Q_{T} \\
\rho, \rho_{0}, \rho_{1}, \rho_{2} \in L^{\infty}\left(Q_{T-\delta}\right) \quad \forall \delta>0 .
\end{array}\right.
$$

Precisely, we will take $\rho_{0}=(T-t)^{3 / 2} \rho, \rho_{1}=(T-t) \rho, \rho_{2}=(T-t)^{1 / 2} \rho$ and $\rho_{3}=(T-t)^{-1 / 2} \rho$ where $\rho$ is defined as follow

$$
\rho(x, t)=\exp \left(\frac{s \beta(x)}{\ell(t)}\right), \quad s \geq C\left(\Omega, \omega, T,\left\|g^{\prime}\right\|_{\infty}\right)
$$

with

$$
\ell(t)= \begin{cases}3 T^{2} / 16 & \text { if } 0 \leq t<T / 4 \\ t(T-t) & \text { if } T / 4 \leq t \leq T\end{cases}
$$

Here $\beta(x)=\exp \left(2 \lambda m\left\|\eta^{0}\right\|_{\infty}\right)-\exp \left(\lambda\left(m\left\|\eta^{0}\right\|_{\infty}+\eta^{0}(x)\right)\right), m>1, \eta^{0} \in \mathcal{C}(\bar{\Omega})$ satisfies $\eta^{0}>0$ in $\Omega, \eta^{0}=0$ on $\partial \Omega$ and $\left|\nabla \eta^{0}\right|>0$ in $\overline{\Omega \backslash \omega}$ (see [12], Lem. 1.2, p. 1401).

In the next section, we shall make use of the following controllability result where $L^{2}(\rho, A):=\{f: \rho f \in$ $\left.L^{2}(A)\right\}$ for any set $A \subset Q_{T}$ and function $\rho$.

Proposition 2.1. Assume $A \in L^{\infty}\left(Q_{T}\right), \rho_{2} B \in L^{2}\left(0, T ; H^{-1}(\Omega)\right)$ and $z_{0} \in L^{2}(\Omega)$. Then there exists a control $v \in L^{2}\left(\rho_{0}, q_{T}\right)$ such that the weak solution $z$ of

$$
\left\{\begin{array}{l}
\partial_{t} z-\Delta z+A z=v 1_{\omega}+B \quad \text { in } \quad Q_{T} \\
z=0 \quad \text { on } \quad \Sigma_{T}, \quad z(\cdot, 0)=z_{0} \quad \text { in } \Omega
\end{array}\right.
$$

satisfies

$$
z(\cdot, T)=0 \text { in } \Omega
$$

Moreover, the unique control $u$ which minimizes together with the corresponding solution $z$ the functional $J$ : $L^{2}\left(\rho, Q_{T}\right) \times L^{2}\left(\rho_{0}, q_{T}\right) \rightarrow \mathbb{R}^{+}$defined by $J(z, v):=\frac{1}{2}\|\rho z\|_{L^{2}\left(Q_{T}\right)}^{2}+\frac{1}{2}\left\|\rho_{0} v\right\|_{L^{2}\left(q_{T}\right)}^{2}$ satisfy the following estimate

$$
\|\rho z\|_{L^{2}\left(Q_{T}\right)}+\left\|\rho_{0} v\right\|_{L^{2}\left(q_{T}\right)} \leq C\left(\left\|\rho_{2} B\right\|_{L^{2}\left(0, T ; H^{-1}(\Omega)\right)}+\left\|z_{0}\right\|_{L^{2}(\Omega)}\right)
$$

for some constant $C=C\left(s, \Omega, \omega, T,\|A\|_{\infty}\right)$.

The controlled solution also satisfies, for some constant $C=C\left(s, \Omega, \omega, T,\|A\|_{\infty}\right)$, the estimate

$$
\left\|\rho_{1} z\right\|_{L^{\infty}\left(0, T ; L^{2}(\Omega)\right)}+\left\|\rho_{1} \nabla z\right\|_{L^{2}\left(Q_{T}\right)^{d}} \leq C\left(\left\|\rho_{2} B\right\|_{L^{2}\left(0, T ; H^{-1}(\Omega)\right)}+\left\|z_{0}\right\|_{L^{2}(\Omega)}\right) .
$$

Proposition 2.1 follows from several lemmas. Let us first set $P_{0}:=\left\{q \in C^{2}\left(\overline{Q_{T}}\right): q=0\right.$ on $\left.\Sigma_{T}\right\}$. The bilinear form

$$
(p, q)_{P}:=\int_{Q_{T}} \rho^{-2} L_{A}^{\star} p L_{A}^{\star} q+\int_{q_{T}} \rho_{0}^{-2} p q
$$


where $L_{A}^{\star} q:=-\partial_{t} q-\Delta q+A q$, is a scalar product on $P_{0}$ (see [14]). The completion $P$ of $P_{0}$ for the norm $\|\cdot\|_{P}$ associated to this scalar product is a Hilbert space and the following result proved in [17] holds.

Lemma 2.2. There exists $C=C\left(s, \Omega, \omega, T,\|A\|_{\infty}\right)>0$ such that one has the following Carleman estimate:

$$
\int_{Q_{T}}\left(\rho_{3}^{-1}\left|p_{t}\right|^{2}+\rho_{2}^{-2}|\nabla p|^{2}+\rho_{0}^{-2}|p|^{2}\right) \leq C\|p\|_{P}^{2}, \quad \forall p \in P .
$$

Remark 2.3. We denote by $P$ (instead of $\left.P_{A}\right)$ the completion of $P_{0}$ for the norm $\|\cdot\|_{P}$ since $P$ does not depend on $A$ (see [13], Lem. 3.1).

Lemma 2.4. There exists $C=C\left(s, \Omega, \omega, T,\|A\|_{\infty}\right)>0$ such that one has the following observability inequality:

$$
\|p(\cdot, 0)\|_{L^{2}(\Omega)} \leq C\|p\|_{P}, \quad \forall p \in P .
$$

Proof. From the definition of $\rho_{0}, \rho_{1}$ and $\rho_{2}, P \hookrightarrow H^{1}\left(0, \frac{T}{2} ; L^{2}(\Omega)\right) \hookrightarrow C\left(\left[0, \frac{T}{2}\right] ; L^{2}(\Omega)\right)$ where each embedding is continuous. The result follows from Lemma 2.2.

Lemma 2.5. There exists $p \in P$ unique solution of

$$
(p, q)_{P}=\int_{\Omega} z_{0} q(0)+\int_{0}^{T}\left\langle\rho_{2} B, \rho_{2}^{-1} q\right\rangle_{H^{-1}(\Omega) \times H_{0}^{1}(\Omega)}, \quad \forall q \in P .
$$

This solution satisfies the following estimate:

$$
\|p\|_{P} \leq C\left(\left\|\rho_{2} B\right\|_{L^{2}\left(0, T ; H^{-1}(\Omega)\right)}+\left\|z_{0}\right\|_{L^{2}(\Omega)}\right)
$$

where $C=C\left(s, \Omega, \omega, T,\|A\|_{\infty}\right)>0$.

Proof. The linear map $L_{1}: P \rightarrow \mathbb{R}, q \mapsto \int_{0}^{T}\left\langle\rho_{2} B, \rho_{2}^{-1} q\right\rangle_{H^{-1}(\Omega) \times H_{0}^{1}(\Omega)}$ is continuous. Indeed, for all $q \in P$

$$
\left|\int_{0}^{T}\left\langle\rho_{2} B, \rho_{2}^{-1} q\right\rangle_{H^{-1}(\Omega) \times H_{0}^{1}(\Omega)}\right| \leq\left(\int_{0}^{T}\left\|\rho_{2} B\right\|_{H^{-1}(\Omega)}^{2}\right)^{1 / 2}\left(\int_{0}^{T}\left\|\rho_{2}^{-1} q\right\|_{H_{0}^{1}(\Omega)}^{2}\right)^{1 / 2}
$$

and a.e. in $(0, T)\left\|\rho_{2}^{-1} q\right\|_{H_{0}^{1}(\Omega)}^{2}=\left\|\rho_{2}^{-1} q\right\|_{L^{2}(\Omega)}^{2}+\left\|\nabla\left(\rho_{2}^{-1} q\right)\right\|_{L^{2}(\Omega)^{d}}^{2}$. But since $\rho_{0} \leq T \rho_{2}$ a.e. $t$ in $(0, T)$

$$
\left\|\rho_{2}^{-1} q\right\|_{L^{2}(\Omega)}^{2} \leq \frac{1}{T^{2}}\left\|\rho_{0}^{-1} q\right\|_{L^{2}(\Omega)}^{2}, \text { a.e. } t \in(0, T) .
$$

Moreover

$$
\nabla\left(\rho_{2}^{-1} q\right)=\nabla\left(\rho_{2}^{-1}\right) q+\rho_{2}^{-1} \nabla q=-\frac{s \nabla \beta(x)}{\ell(t)(T-t)^{1 / 2}} \rho^{-1} q+\rho_{2}^{-1} \nabla q
$$

and thus, since $\rho_{1} \leq T^{1 / 2} \rho_{2}$ a.e. $t$ in $(0, T)$ :

$$
\begin{aligned}
\left\|\nabla\left(\rho_{2}^{-1} q\right)\right\|_{L^{2}(\Omega)^{d}}^{2} & \leq\left\|\frac{s \nabla \beta(x)}{\ell(t)(T-t)^{1 / 2}} \rho^{-1} q\right\|_{L^{2}(\Omega)^{d}}^{2}+\left\|\rho_{2}^{-1} \nabla q\right\|_{L^{2}(\Omega)^{d}}^{2} \\
& \leq C\left(s, \Omega, \omega, T,\|A\|_{\infty}\right)\left(\left\|\rho_{0}^{-1} q\right\|_{L^{2}(\Omega)}^{2}+\left\|\rho_{2}^{-1} \nabla q\right\|_{L^{2}(\Omega)^{d}}^{2}\right) .
\end{aligned}
$$


We then deduce that, a.e. in $(0, T)$

$$
\left\|\rho_{2}^{-1} q\right\|_{H_{0}^{1}(\Omega)}^{2} \leq C\left(s, \Omega, \omega, T,\|A\|_{\infty}\right)\left(\left\|\rho_{0}^{-1} q\right\|_{L^{2}(\Omega)}^{2}+\left\|\rho_{1}^{-1} \nabla q\right\|_{L^{2}(\Omega)^{d}}^{2}\right)
$$

and from the Carleman estimate (2.7) that

$$
\left(\int_{0}^{T}\left\|\rho_{2}^{-1} q\right\|_{H_{0}^{1}(\Omega)}^{2}\right)^{1 / 2} \leq C\left(s, \Omega, \omega, T,\|A\|_{\infty}\right)\|q\|_{P}
$$

and therefore

$$
\left|\int_{0}^{T}\left\langle\rho_{2} B, \rho_{2}^{-1} q\right\rangle_{H^{-1}(\Omega) \times H_{0}^{1}(\Omega)}\right| \leq C\left(s, \Omega, \omega, T,\|A\|_{\infty}\right)\left(\int_{0}^{T}\left\|\rho_{2} B\right\|_{H^{-1}(\Omega)}^{2}\right)^{1 / 2}\|q\|_{P} .
$$

Thus $L_{1}$ is continuous.

From (2.8) we easily deduce that the linear map $L_{2}: P \rightarrow \mathbb{R}, q \mapsto \int_{\Omega} z_{0} q(0)$ is continuous. Using Riesz's theorem, we conclude that there exists exactly one solution $p \in P$ of $(2.9)$.

Let us now introduce the convex set

$$
C\left(z_{0}, T\right)=\left\{(z, v): \rho z \in L^{2}\left(Q_{T}\right), \rho_{0} v \in L^{2}\left(q_{T}\right),(z, v) \text { solves }(2.3)-(2.4) \text { in the transposition sense }\right\}
$$

that is $(z, v)$ is solution of

$$
\int_{Q_{T}} z L_{A}^{\star} q=\int_{q_{T}} v q+\int_{\Omega} z_{0} q(0)+\int_{0}^{T}\langle B, q\rangle_{H^{-1}(\Omega) \times H_{0}^{1}(\Omega)}, \quad \forall q \in P .
$$

Let us remark that if $(z, v) \in C\left(z_{0}, T\right)$, then since $z_{0} \in L^{2}(\Omega), v \in L^{2}\left(q_{T}\right)$ and $B \in L^{2}\left(0, T ; H^{-1}(\Omega)\right), z$ must coincide with the unique weak solution of (2.3) associated with $v$.

We can now claim that $C\left(z_{0}, T\right)$ is a non empty. Indeed we have:

Lemma 2.6. Let $p \in P$ defined in Lemma 2.5 and $(z, v)$ defined by

$$
z=\rho^{-2} L_{A}^{\star} p \quad \text { and } \quad v=-\left.\rho_{0}^{-2} p\right|_{q_{T}} .
$$

Then $(z, v) \in C\left(z_{0}, T\right)$ and satisfies the following estimate

$$
\|\rho z\|_{L^{2}\left(Q_{T}\right)}+\left\|\rho_{0} v\right\|_{L^{2}\left(q_{T}\right)} \leq C\left(\left\|\rho_{2} B\right\|_{L^{2}\left(0, T ; H^{-1}(\Omega)\right)}+\left\|z_{0}\right\|_{L^{2}(\Omega)}\right)
$$

where $C=C\left(s, \Omega, \omega, T,\|A\|_{\infty}\right)>0$.

Proof. Let us prove that $(z, v)$ belongs to $C\left(z_{0}, T\right)$. From the definition of $P, \rho z \in L^{2}\left(Q_{T}\right)$ and $\rho_{0} v \in L^{2}\left(q_{T}\right)$ and from the definition of $\rho, \rho_{0}, \rho_{2}, z \in L^{2}\left(Q_{T}\right)$ and $v \in L^{2}\left(q_{T}\right)$. In view of $(2.9),(z, v)$ is solution of

$$
\int_{Q_{T}} z L_{A}^{\star} q=\int_{q_{T}} v q+\int_{\Omega} z_{0} q(0)+\int_{0}^{T}\left\langle\rho_{2} B, \rho_{2}^{-1} q\right\rangle_{H^{-1}(\Omega) \times H_{0}^{1}(\Omega)}, \quad \forall q \in P
$$


that is, since from the definition of $\rho_{2}, B \in L^{2}\left(0, T ; H^{-1}(\Omega)\right)$ and

$$
\int_{0}^{T}\left\langle\rho_{2} B, \rho_{2}^{-1} q\right\rangle_{H^{-1}(\Omega) \times H_{0}^{1}(\Omega)}=\int_{0}^{T}\langle B, q\rangle_{H^{-1}(\Omega) \times H_{0}^{1}(\Omega)},
$$

$z$ is the solution of (2.3) associated with $v$ in the transposition sense. Thus $C\left(z_{0}, T\right) \neq \emptyset$.

Let us now consider the following extremal problem, introduced by Fursikov and Imanuvilov [17]

$$
\left\{\begin{array}{l}
\text { Minimize } J(z, v)=\frac{1}{2}\|(z, v)\|_{L^{2}\left(\rho, Q_{T}\right) \times L^{2}\left(\rho_{0}, q_{T}\right)}^{2}=\frac{1}{2} \int_{Q_{T}} \rho^{2}|z|^{2}+\frac{1}{2} \int_{q_{T}} \rho_{0}^{2}|v|^{2} \\
\text { subject to }(z, v) \in C\left(z_{0}, T\right) .
\end{array}\right.
$$

Then $(z, v) \mapsto J(z, v)$ is clearly strictly convex and continuous on $L^{2}\left(\rho, Q_{T}\right) \times L^{2}\left(\rho_{0}, q_{T}\right)$. Therefore $(2.12)$ possesses at most a unique solution in $C\left(z_{0}, T\right)$. More precisely we have:

Proposition 2.7. $(z, v) \in C\left(z_{0}, T\right)$ defined in Lemma 2.6 is the unique solution of (2.12).

Proof. Let $(y, w) \in C\left(z_{0}, T\right)$. Since $J$ is convex and differentiable on $L^{2}\left(\rho, Q_{T}\right) \times L^{2}\left(\rho_{0}, q_{T}\right)$ we have:

$$
\begin{aligned}
J(y, w) & \geq J(z, v)+\int_{Q_{T}} \rho^{2} z(y-z)+\int_{q_{T}} \rho_{0}^{2} v(w-v) \\
& =J(z, v)+\int_{Q_{T}} L^{\star} p(y-z)-\int_{q_{T}} p(w-v)=J(z, v)
\end{aligned}
$$

$y$ being the solution of (2.3) associated with $w$ in the transposition sense. Hence $(z, v)$ solves $(2.12)$.

Proof. (of Prop. 2.1) It suffices to prove that $(z, v)$ satisfies the estimate (2.6). Since $z$ is a weak solution of (2.3) associated with $v, z \in L^{2}\left(0, T ; H_{0}^{1}(\Omega)\right)$ and $z_{t} \in L^{2}\left(0, T ; H^{-1}(\Omega)\right)$. Multiplying (2.3) by $\rho_{1}^{2} z$ and integrating by part we obtain, a.e. $t$ in $(0, T)$

$$
\begin{gathered}
\frac{1}{2} \partial_{t} \int_{\Omega}|z|^{2} \rho_{1}^{2}-\int_{\Omega}|z|^{2} \rho_{1} \partial_{t} \rho_{1}+\int_{\Omega} \rho_{1}^{2}|\nabla z|^{2}+2 \int_{\Omega} \rho_{1} z \nabla \rho_{1} \cdot \nabla z+\int_{\Omega} \rho_{1}^{2} A z z \\
=\int_{\omega} v \rho_{1}^{2} z+\left\langle B, \rho_{1}^{2} z\right\rangle_{H^{-1}(\Omega) \times H_{0}^{1}(\Omega)} .
\end{gathered}
$$

But $\partial_{t} \rho_{1}=-\rho-(T-t) \frac{s \beta \ell^{\prime}(t)}{\ell(t)^{2}} \rho$, so that

$$
\left.\left.\left|\int_{\Omega}\right| z\right|^{2} \rho_{1} \partial_{t} \rho_{1}\left|\leq C\left(s, \Omega, \omega, T,\|A\|_{\infty}\right) \int_{\Omega}\right| \rho z\right|^{2} .
$$

Since $\nabla \rho_{1}=(T-t) \nabla \rho=(T-t) \frac{s \nabla \beta}{\ell(t)} \rho$ and $\frac{T-t}{l(t)} \leq C(T)$, we have

$$
\left|\int_{\Omega} \rho_{1} z \nabla \rho_{1} \cdot \nabla z\right| \leq C\left(s, \Omega, \omega, T,\|A\|_{\infty}\right)\left(\int_{\Omega}\left|\rho_{1} \nabla z\right|^{2}\right)^{1 / 2}\left(\int_{\Omega}|\rho z|^{2}\right)^{1 / 2} .
$$

The following estimates also hold

$$
\left|\int_{\Omega} \rho_{1}^{2} A z z\right| \leq C\left(T,\|A\|_{\infty}\right) \int_{\Omega}|\rho z|^{2}
$$




$$
\left|\int_{\omega} v \rho_{1}^{2} z\right| \leq T^{1 / 2}\left|\int_{\omega} \rho_{0} v \rho z\right| \leq T^{1 / 2}\left(\int_{\omega}\left|\rho_{0} v\right|^{2}\right)^{1 / 2}\left(\int_{\Omega}|\rho z|^{2}\right)^{1 / 2}
$$

and

$$
\begin{aligned}
\left|\left\langle B, \rho_{1}^{2} z\right\rangle_{H^{-1}(\Omega) \times H_{0}^{1}(\Omega)}\right| & =\left|\left\langle\rho_{1} B, \rho_{1} z\right\rangle_{H^{-1}(\Omega) \times H_{0}^{1}(\Omega)}\right| \leq\left\|\rho_{1} B\right\|_{H^{-1}(\Omega)}\left\|\rho_{1} z\right\|_{H_{0}^{1}(\Omega)} \\
& \leq C\left(s, \Omega, \omega, T,\|A\|_{\infty}\right)\left\|\rho_{2} B\right\|_{H^{-1}(\Omega)}\left(\|\rho z\|_{L^{2}(\Omega)}+\left\|\rho_{1} \nabla z\right\|_{L^{2}(\Omega)^{d}}\right) .
\end{aligned}
$$

Thus we easily obtain that

$$
\partial_{t} \int_{\Omega} \rho_{1}^{2}|z|^{2}+\int_{\Omega} \rho_{1}^{2}|\nabla z|^{2} \leq C\left(s, \Omega, \omega, T,\|A\|_{\infty}\right)\left(\left\|\rho_{2} B\right\|_{H^{-1}(\Omega)}^{2}+\int_{\Omega} \rho^{2}|z|^{2}+\int_{\omega}\left|\rho_{0} v\right|^{2}\right)
$$

and therefore, using (2.11), for all $t \in[0, T]$ :

$$
\left(\int_{\Omega} \rho_{1}^{2}|z|^{2}\right)(t)+\int_{Q_{t}} \rho_{1}^{2}|\nabla z|^{2} \leq C\left(s, \Omega, \omega, T,\|A\|_{\infty}\right)\left(\left\|\rho_{2} B\right\|_{L^{2}\left(0, T ; H^{-1}(\Omega)\right)}^{2}+\left\|z_{0}\right\|_{L^{2}(\Omega)}^{2}\right)
$$

which gives (2.6) and concludes the proof of Proposition 2.1.

\section{THE LEAST-SQUARES METHOD AND ITS ANALYSIS}

For any $p \in[0,1]$, we define the space

$$
W_{p}=\left\{g \in \mathcal{C}(\mathbb{R}), g(0)=0, g^{\prime} \in L^{\infty}(\mathbb{R}),\left[g^{\prime}\right]_{p}:=\sup _{a, b \in \mathbb{R}, a \neq b} \frac{\left|g^{\prime}(a)-g^{\prime}(b)\right|}{|a-b|^{p}}<\infty\right\} .
$$

The case $p=0$ reduces to $W_{0}=\left\{g \in \mathcal{C}(\mathbb{R}), g(0)=0, g^{\prime} \in L^{\infty}(\mathbb{R})\right\}$ with $\left[g^{\prime}\right]_{0}=2\left\|g^{\prime}\right\|_{\infty}$. The case $p=1$ corresponds to $W_{1}=\left\{g \in \mathcal{C}(\mathbb{R}), g(0)=0, g^{\prime} \in L^{\infty}(\mathbb{R}), g^{\prime \prime} \in L^{\infty}(\mathbb{R})\right\}$ with $\left[g^{\prime}\right]_{1}=\left\|g^{\prime \prime}\right\|_{\infty}$. The property $\left[g^{\prime}\right]_{p}<$ $\infty$ means that $g^{\prime}$ is uniformly Hölder continuous with exponent $p$.

In the sequel, we shall assume that there exists some $p \in(0,1]$ for which the nonlinear function $g$ belongs to $W_{p}$. Remark that $g \in W_{p}$ for some $p \in[0,1]$ satisfies hypotheses (1.2) and (1.6). We shall also assume that $u_{0} \in L^{2}(\Omega)$.

\subsection{The least-squares method}

We introduce the vectorial space $\mathcal{A}_{0}$ as follows

$$
\begin{aligned}
\mathcal{A}_{0}=\{(y, f): & \rho y \in L^{2}\left(Q_{T}\right), \rho_{1} \nabla y \in L^{2}\left(Q_{T}\right)^{d}, \rho_{0} f \in L^{2}\left(q_{T}\right), \\
& \left.\rho_{2}\left(\partial_{t} y-\Delta y-f 1_{\omega}\right) \in L^{2}\left(0, T ; H^{-1}(\Omega)\right), y(\cdot, 0)=0 \text { in } \Omega, y=0 \text { on } \Sigma_{T}\right\}
\end{aligned}
$$

where $\rho, \rho_{2}, \rho_{1}$ and $\rho_{0}$ are defined in $(2.2)$. Since $L^{2}\left(0, T ; H^{-1}(\Omega)\right)$ is also a Hilbert space, $\mathcal{A}_{0}$ endowed with the following scalar product

$$
\begin{aligned}
((y, f),(\bar{y}, \bar{f}))_{\mathcal{A}_{0}}=(\rho y, \rho \bar{y})_{2} & +\left(\rho_{1} \nabla y, \rho_{1} \nabla \bar{y}\right)_{2}+\left(\rho_{0} f, \rho_{0} \bar{f}\right)_{2} \\
& +\left(\rho_{2}\left(\partial_{t} y-\Delta y-f 1_{\omega}\right), \rho_{2}\left(\partial_{t} \bar{y}-\Delta \bar{y}-\bar{f} 1_{\omega}\right)\right)_{L^{2}\left(0, T ; H^{-1}(\Omega)\right)}
\end{aligned}
$$


is a Hilbert space. The corresponding norm is $\|(y, f)\|_{\mathcal{A}_{0}}=\sqrt{((y, f),(y, f))_{\mathcal{A}_{0}}}$. We also consider the convex space

$$
\begin{aligned}
\mathcal{A}=\{(y, f): & \rho y \in L^{2}\left(Q_{T}\right), \rho_{1} \nabla y \in L^{2}\left(Q_{T}\right)^{d}, \rho_{0} f \in L^{2}\left(q_{T}\right), \\
& \left.\rho_{2}\left(\partial_{t} y-\Delta y-f 1_{\omega}\right) \in L^{2}\left(0, T ; H^{-1}(\Omega)\right), y(\cdot, 0)=u_{0} \text { in } \Omega, y=0 \text { on } \Sigma_{T}\right\}
\end{aligned}
$$

so that we can write $\mathcal{A}=(\bar{y}, \bar{f})+\mathcal{A}_{0}$ for any element $(\bar{y}, \bar{f}) \in \mathcal{A}$. We endow $\mathcal{A}$ with the same norm. Clearly, if $(y, f) \in \mathcal{A}$, then $y \in C\left([0, T] ; L^{2}(\Omega)\right)$ and since $\rho y \in L^{2}\left(Q_{T}\right)$, then $y(\cdot, T)=0$. The null controllability requirement is therefore incorporated in the spaces $\mathcal{A}_{0}$ and $\mathcal{A}$.

For any fixed $(\bar{y}, \bar{f}) \in \mathcal{A}$, we can now consider the following non convex extremal problem:

$$
\inf _{(y, f) \in \mathcal{A}_{0}} E(\bar{y}+y, f+\bar{f})
$$

where $E: \mathcal{A} \rightarrow \mathbb{R}$ is defined as follows

$$
E(y, f):=\frac{1}{2}\left\|\rho_{2}\left(\partial_{t} y-\Delta y+g(y)-f 1_{\omega}\right)\right\|_{L^{2}\left(0, T ; H^{-1}(\Omega)\right)}^{2}
$$

justifying the least-squares terminology we have used.

Let us remark that, if $g \in W_{p}$ for one $p \geq 0$, then $g$ is Lipschitz and thus, since $g(0)=0$, there exists $K>0$ such that $|g(\xi)| \leq K|\xi|$ for all $\xi \in \mathbb{R}$. Consequently, $\rho_{2} g(y) \in L^{2}\left(Q_{T}\right)$ (and then $\rho_{2} g(y) \in L^{2}\left(0, T ; H^{-1}(\Omega)\right)$ ) since

$$
\left\|\rho_{2} g(y)\right\|_{L^{2}\left(Q_{T}\right)}=\left\|\left(\rho_{2} \rho^{-1}\right) \rho g(y)\right\|_{L^{2}\left(Q_{T}\right)}=\left\|(T-t)^{1 / 2} \rho g(y)\right\|_{L^{2}\left(Q_{T}\right)} \leq T^{1 / 2} K\|\rho y\|_{L^{2}\left(Q_{T}\right)} .
$$

Any pair $(y, f) \in \mathcal{A}$ for which $E(y, f)$ vanishes is a controlled pair of (1.1), and conversely. In this sense, the functional $E$ is a so-called error functional which measures the deviation of $(y, f)$ from being a solution of the underlying nonlinear equation. Although any $g \in W_{p}$ for some $p \geq 0$ satisfies hypotheses (1.2) and (1.6), the controllability result of Theorem 1.2 given in [16] does not imply the existence of zero of $E$ in $\mathcal{A}$, since controls of minimal $L^{\infty}\left(q_{T}\right)$ norm are considered there. Our constructive approach will show that the extremal problem (3.2) admits solutions.

We also emphasize that the $L^{2}\left(0, T ; H^{-1}(\Omega)\right)$ norm in $E$ indicates that we are looking for weak solutions of the parabolic equation (1.1). We refer to $[22,23]$ where a similar so-called weak least-squares method is employed to approximate the solutions of the unsteady Navier-Stokes equation.

A practical way of taking a functional to its minimum is through some clever use of descent directions, i.e the use of its derivative. In doing so, the presence of local minima is always something that may dramatically spoil the whole scheme. The unique structural property that discards this possibility is the strict convexity of the functional $E$. However, for nonlinear equation like (1.1), one cannot expect this property to hold for the functional $E$. Nevertheless, we insist in that one may construct a particular minimizing sequence which cannot converge except to a global minimizer leading $E$ down to zero.

In order to construct such minimizing sequence, we look, for any $(y, f) \in \mathcal{A}$, for a pair $\left(Y^{1}, F^{1}\right) \in \mathcal{A}_{0}$ solution of the following formulation

$$
\left\{\begin{array}{l}
\partial_{t} Y^{1}-\Delta Y^{1}+g^{\prime}(y) Y^{1}=F^{1} 1_{\omega}+\partial_{t} y-\Delta y+g(y)-f 1_{\omega} \text { in } Q_{T}, \\
Y^{1}=0 \quad \text { on } \quad \Sigma_{T}, \quad Y^{1}(\cdot, 0)=0 \quad \text { in } \Omega .
\end{array}\right.
$$


Since $\left(Y^{1}, F^{1}\right) \in \mathcal{A}_{0}, F^{1}$ is a null control for $Y^{1}$. We have the following property.

Proposition 3.1. Let any $(y, f) \in \mathcal{A}$. There exists a pair $\left(Y^{1}, F^{1}\right) \in \mathcal{A}_{0}$ solution of (3.3) which satisfies the following estimate:

$$
\left\|\left(Y^{1}, F^{1}\right)\right\|_{\mathcal{A}_{0}} \leq C \sqrt{E(y, f)}
$$

for some $C=C\left(s, \Omega, \omega, T,\left\|g^{\prime}\right\|_{\infty}\right)>0$.

Proof. For all $(y, f) \in \mathcal{A}$ we have $\rho_{2}\left(\partial_{t} y-\Delta y+g(y)-f 1_{\omega}\right) \in L^{2}\left(0, T ; H^{-1}(\Omega)\right)$. The existence of a null control $F^{1}$ is therefore given by Proposition 2.1. Choosing the control $F^{1}$ which minimizes together with the corresponding solution $Y^{1}$ the functional $J$ defined in Proposition 2.1, we get the following estimate (since $\left.Y^{1}(\cdot, 0)=0\right)$

$$
\begin{aligned}
\left\|\rho Y^{1}\right\|_{L^{2}\left(Q_{T}\right)}+\left\|\rho_{0} F^{1}\right\|_{L^{2}\left(q_{T}\right)} & \leq C\left\|\rho_{2}\left(\partial_{t} y-\Delta y+g(y)-f 1_{\omega}\right)\right\|_{L^{2}\left(0, T ; H^{-1}(\Omega)\right)} \\
& \leq C \sqrt{E(y, f)}
\end{aligned}
$$

and

$$
\begin{aligned}
\left\|\rho_{1} Y^{1}\right\|_{L^{\infty}\left(0, T ; L^{2}(\Omega)\right)}+\left\|\rho_{1} \nabla Y^{1}\right\|_{L^{2}\left(Q_{T}\right)^{d}} & \leq C\left\|\rho_{2}\left(\partial_{t} y-\Delta y+g(y)-f 1_{\omega}\right)\right\|_{L^{2}\left(0, T ; H^{-1}(\Omega)\right)} \\
& \leq C \sqrt{E(y, f)}
\end{aligned}
$$

for some $C=C\left(s, \Omega, \omega, T,\|g\|_{\infty}\right)$ independent of $Y^{1}, F^{1}$ and $y$. Eventually, from the equation solved by $Y^{1}$,

$$
\begin{aligned}
\left\|\rho_{2}\left(\partial_{t} Y^{1}-\Delta Y^{1}-F^{1} 1_{\omega}\right)\right\|_{L^{2}\left(0, T ; H^{-1}(\Omega)\right)} & \leq\left\|\rho_{2} g^{\prime}(y) Y^{1}\right\|_{L^{2}\left(Q_{T}\right)}+\left\|\rho_{2}\left(\partial_{t} y-\Delta y+g(y)-f 1_{\omega}\right)\right\|_{L^{2}\left(0, T ; H^{-1}(\Omega)\right)} \\
& \leq\left\|(T-t)^{1 / 2} g^{\prime}(y)\right\|_{\infty}\left\|\rho Y^{1}\right\|_{L^{2}\left(Q_{T}\right)}+\sqrt{2 E(y, f)} \\
& \leq \max \left(1,\left\|(T-t)^{1 / 2} g^{\prime}\right\|_{\infty}\right) C \sqrt{E(y, f)}
\end{aligned}
$$

which proves that $\left(Y^{1}, F^{1}\right)$ belongs to $\mathcal{A}_{0}$.

Remark 3.2. From (3.3), we check that $z=y-Y^{1}$ is a null controlled solution satisfying

$$
\left\{\begin{array}{l}
\partial_{t} z-\Delta z+g^{\prime}(y) z=\left(f-F^{1}\right) 1_{\omega}+g^{\prime}(y) y-g(y) \quad \text { in } \quad Q_{T}, \\
z=0 \text { on } \Sigma_{T}, \quad z(\cdot, 0)=u_{0} \quad \text { in } \Omega
\end{array}\right.
$$

by the control $\left(f-F^{1}\right) \in L^{2}\left(\rho_{0}, q_{T}\right)$.

Remark 3.3. We emphasize that the presence of a right hand side in (3.3), namely $y_{t}-\Delta y+g(y)-f 1_{\omega}$, forces us to introduce the weights $\rho_{0}, \rho_{1}, \rho_{2}$ and $\rho$ in the spaces $\mathcal{A}_{0}$ and $\mathcal{A}$. This can be seen from the equality (2.9): since $\rho_{2}^{-1} q$ belongs to $L^{2}\left(0, T ; H^{1}(\Omega)\right)$ for all $q \in P$, we need to impose that $\rho_{2} B \in L^{2}\left(0, T ; H^{-1}(\Omega)\right)$ with here $B=\partial_{t} y-\Delta y+g(y)-f 1_{\omega}$. Working with the linearized equation (1.7) (introduced in [16]) which does not make appear an additional right hand side, we may avoid the introduction of Carleman type weights. Actually, the authors in [16] consider controls of minimal $L^{\infty}\left(q_{T}\right)$ norm. Introduction of weights allows however the characterization (2.9), which is very convenient at the practical level. We refer to [14] where this is discussed at length.

The interest of the pair $\left(Y^{1}, F^{1}\right) \in \mathcal{A}_{0}$ lies in the following result. 
Proposition 3.4. Let $(y, f) \in \mathcal{A}$ and let $\left(Y^{1}, F^{1}\right) \in \mathcal{A}_{0}$ be a solution of (3.3). Then the derivative of $E$ at the point $(y, f) \in \mathcal{A}$ along the direction $\left(Y^{1}, F^{1}\right)$ given by $E^{\prime}(y, f) \cdot\left(Y^{1}, F^{1}\right):=\lim _{\eta \rightarrow 0, \eta \neq 0} \frac{E\left((y, f)+\eta\left(Y^{1}, F^{1}\right)\right)-E(y, f)}{\eta}$ satisfies

$$
E^{\prime}(y, f) \cdot\left(Y^{1}, F^{1}\right)=2 E(y, f) .
$$

Proof. We preliminary check that for all $(Y, F) \in \mathcal{A}_{0}, E$ is differentiable at the point $(y, f) \in \mathcal{A}$ along the direction $(Y, F) \in \mathcal{A}_{0}$. For all $\lambda \in \mathbb{R}$, simple computations lead to the equality

$$
E(y+\lambda Y, f+\lambda F)=E(y, f)+\lambda E^{\prime}(y, f) \cdot(Y, F)+h((y, f), \lambda(Y, F))
$$

with

$$
E^{\prime}(y, f) \cdot(Y, F):=\left(\rho_{2}\left(\partial_{t} y-\Delta y+g(y)-f 1_{\omega}\right), \rho_{2}\left(\partial_{t} Y-\Delta Y+g^{\prime}(y) Y-F 1_{\omega}\right)\right)_{L^{2}\left(0, T ; H^{-1}(\Omega)\right)}
$$

and

$$
\begin{aligned}
h((y, f), \lambda(Y, F))= & \lambda\left(\rho_{2}\left(\partial_{t} Y-\Delta Y+g^{\prime}(y) Y-F 1_{\omega}\right), \rho_{2} l(y, \lambda Y)\right)_{L^{2}\left(0, T ; H^{-1}(\Omega)\right)} \\
& +\frac{\lambda^{2}}{2}\left\|\rho_{2}\left(\partial_{t} Y-\Delta Y+g^{\prime}(y) Y-F 1_{\omega}\right)\right\|_{L^{2}\left(0, T ; H^{-1}(\Omega)\right)}^{2} \\
& +\left(\rho_{2}\left(\partial_{t} y-\Delta y+g(y)-f 1_{\omega}\right), \rho_{2} l(y, \lambda Y)\right)_{L^{2}\left(0, T ; H^{-1}(\Omega)\right)} \\
& +\frac{1}{2}\left\|\rho_{2} l(y, \lambda Y)\right\|_{L^{2}\left(0, T ; H^{-1}(\Omega)\right)}^{2}
\end{aligned}
$$

where $l(y, \lambda Y)=g(y+\lambda Y)-g(y)-\lambda g^{\prime}(y) Y$.

The application $(Y, F) \rightarrow E^{\prime}(y, f) \cdot(Y, F)$ is linear and continuous from $\mathcal{A}_{0}$ to $\mathbb{R}$ as it satisfies

$$
\begin{aligned}
& \left|E^{\prime}(y, f) \cdot(Y, F)\right| \\
& \quad \leq\left\|\rho_{2}\left(\partial_{t} y-\Delta y+g(y)-f 1_{\omega}\right)\right\|_{L^{2}\left(0, T ; H^{-1}(\Omega)\right)}\left\|\rho_{2}\left(\partial_{t} Y-\Delta Y+g^{\prime}(y) Y-F 1_{\omega}\right)\right\|_{L^{2}\left(0, T ; H^{-1}(\Omega)\right)} \\
& \quad \leq \sqrt{2 E(y, f)}\left(\left\|\rho_{2}\left(\partial_{t} Y-\Delta Y-F 1_{\omega}\right)\right\|_{L^{2}\left(0, T ; H^{-1}(\Omega)\right)}+\left\|\rho_{2} g^{\prime}(y) Y\right\|_{L^{2}\left(Q_{T}\right)}\right) \\
& \quad \leq \sqrt{2 E(y, f)}\left(\left\|\rho_{2}\left(\partial_{t} Y-\Delta Y-F 1_{\omega}\right)\right\|_{L^{2}\left(0, T ; H^{-1}(\Omega)\right)}+\left\|(T-t)^{1 / 2} g^{\prime}(y)\right\|_{L^{\infty}\left(Q_{T}\right)}\|\rho Y\|_{L^{2}\left(Q_{T}\right)}\right) \\
& \quad \leq \sqrt{2 E(y, f)} \max \left(1,\left\|(T-t)^{1 / 2} g^{\prime}\right\|_{\infty}\right)\|(Y, F)\|_{\mathcal{A}_{0}} .
\end{aligned}
$$

Similarly, for all $\lambda \in \mathbb{R}^{\star}$

$$
\begin{aligned}
\left|\frac{1}{\lambda} h((y, f), \lambda(Y, F))\right| \leq & \left(\lambda\left\|\rho_{2}\left(\partial_{t} Y-\Delta Y+g^{\prime}(y) Y-F 1_{\omega}\right)\right\|_{L^{2}\left(0, T ; H^{-1}(\Omega)\right)}+\sqrt{2 E(y, f)}\right. \\
& \left.+\frac{1}{2}\left\|\rho_{2} l(y, \lambda Y)\right\|_{L^{2}\left(0, T ; H^{-1}(\Omega)\right)}\right) \frac{1}{\lambda}\left\|\rho_{2} l(y, \lambda Y)\right\|_{L^{2}\left(0, T ; H^{-1}(\Omega)\right)} \\
& +\frac{\lambda}{2}\left\|\rho_{2}\left(\partial_{t} Y-\Delta Y+g^{\prime}(y) Y-F 1_{\omega}\right)\right\|_{L^{2}\left(0, T ; H^{-1}(\Omega)\right)}^{2} .
\end{aligned}
$$


Since $g^{\prime} \in L^{\infty}(\mathbb{R})$ we have for a.e. $(x, t) \in Q_{T}$ :

$$
\rho_{2}\left|\frac{1}{\lambda} l(y, \lambda Y)\right|=\rho_{2}\left|\frac{g(y+\lambda Y)-g(y)}{\lambda}-g^{\prime}(y) Y\right| \leq 2\left\|g^{\prime}\right\|_{\infty}\left|\rho_{2} Y\right|
$$

and $\rho_{2} Y \in L^{2}\left(Q_{T}\right)$. Moreover, for a.e. $(x, t) \in Q_{T}, \rho_{2}\left|\frac{1}{\lambda} l(y, \lambda Y)\right|=\rho_{2}\left|\frac{g(y+\lambda Y)-g(y)}{\lambda}-g^{\prime}(y) Y\right| \rightarrow 0$ as $\lambda \rightarrow 0$; it follows from the Lebesgue's dominated convergence theorem that $\frac{1}{\lambda}\left\|\rho_{2} l(y, \lambda Y)\right\|_{L^{2}\left(Q_{T}\right)} \rightarrow 0$ as $\lambda \rightarrow 0$. It is now easy to see that $h((y, f), \lambda(Y, F))=o(\lambda)$ and that the functional $E$ is differentiable at the point $(y, f) \in \mathcal{A}$ along the direction $(Y, F) \in \mathcal{A}_{0}$. Eventually, the equality (3.9) follows from the definition of the pair $\left(Y^{1}, F^{1}\right)$ given in (3.3).

Remark that from the equality (3.10), the derivative $E^{\prime}(y, f)$ is independent of $(Y, F)$. We can then define the norm $\left\|E^{\prime}(y, f)\right\|_{\left(\mathcal{A}_{0}\right)^{\prime}}:=\sup _{(Y, F) \in \mathcal{A}_{0} \backslash\{0\}} \frac{E^{\prime}(y, f) \cdot(Y, F)}{\|(Y, F)\|_{\mathcal{A}_{0}}}$ associated with $\mathcal{A}_{0}^{\prime}$, the topological dual of $\mathcal{A}_{0}$.

Combining the equality (3.9) and the inequality (3.4), we deduce the following estimates of $E(y, f)$ in term of the norm of $E^{\prime}(y, f)$.

Proposition 3.5. For any $(y, f) \in \mathcal{A}$, the inequalities hold true

$$
C_{1}\left(s, \Omega, \omega, T,\left\|g^{\prime}\right\|_{\infty}\right)\left\|E^{\prime}(y, f)\right\|_{\mathcal{A}_{0}^{\prime}} \leq \sqrt{E(y, f)} \leq C_{2}\left(s, \Omega, \omega, T,\left\|g^{\prime}\right\|_{\infty}\right)\left\|E^{\prime}(y, f)\right\|_{\mathcal{A}_{0}^{\prime}}
$$

for some constants $C_{1}, C_{2}>0$.

Proof. (3.9) rewrites $E(y, f)=\frac{1}{2} E^{\prime}(y, f) \cdot\left(Y^{1}, F^{1}\right)$ where $\left(Y^{1}, F^{1}\right) \in \mathcal{A}_{0}$ is solution of (3.3) and therefore, with

$$
E(y, f) \leq \frac{1}{2}\left\|E^{\prime}(y, f)\right\|_{\mathcal{A}_{0}^{\prime}}\left\|\left(Y^{1}, F^{1}\right)\right\|_{\mathcal{A}_{0}} \leq C\left(s, \Omega, \omega, T,\left\|g^{\prime}\right\|_{\infty}\right)\left\|E^{\prime}(y, f)\right\|_{\mathcal{A}_{0}^{\prime}} \sqrt{E(y, f)} .
$$

On the other hand, for all $(Y, F) \in \mathcal{A}_{0}$ (see the proof of Prop. 3.4):

$$
\left|E^{\prime}(y, f) \cdot(Y, F)\right| \leq \sqrt{2 E(y, f)} \max \left(1,\left\|(T-t)^{1 / 2} g^{\prime}\right\|_{\infty}\right)\|(Y, F)\|_{\mathcal{A}_{0}}
$$

and thus $C_{1}\left(s, \Omega, \omega, T,\left\|g^{\prime}\right\|_{\infty}\right)\left\|E^{\prime}(y, f)\right\|_{\mathcal{A}_{0}^{\prime}} \leq \sqrt{E(y, f)}$.

In particular, any critical point $(y, f) \in \mathcal{A}$ for $E$ (i.e. for which $E^{\prime}(y, f)$ vanishes) is a zero for $E$, a pair solution of the controllability problem. In other words, any sequence $\left(y_{k}, f_{k}\right)_{k>0}$ satisfying $\left\|E^{\prime}\left(y_{k}, f_{k}\right)\right\|_{\mathcal{A}_{0}^{\prime}} \rightarrow 0$ as $k \rightarrow \infty$ is such that $E\left(y_{k}, f_{k}\right) \rightarrow 0$ as $k \rightarrow \infty$. We insist that this property does not imply the convexity of the functional $E$ (and a fortiori the strict convexity of $E$, which actually does not hold here in view of the multiple zeros for $E$ ) but show that a minimizing sequence for $E$ can not be stuck in a local minimum. Far from the zeros of $E$, in particular, when $\|(y, f)\|_{\mathcal{A}} \rightarrow \infty$, the right hand side inequality indicates that $E$ tends to be convex. On the other hand, the left inequality indicates that the functional $E$ is flat around its zero set. As a consequence, gradient based minimizing sequences may achieve a very low rate of convergence (we refer to [26] and also [24] devoted to the Navier-Stokes equation where this phenomenon is observed).

\subsection{A strongly converging minimizing sequence for $E$}

We now examine the convergence of an appropriate sequence $\left(y_{k}, f_{k}\right)_{k \in \mathbb{N}} \in \mathcal{A}$. In this respect, we observe that the equality (3.9) shows that $-\left(Y^{1}, F^{1}\right)$ given by the solution of $(3.3)$ is a descent direction for the functional 
$E$. Therefore, we can define at least formally, for any $m \geq 1$, a minimizing sequence $\left(y_{k}, f_{k}\right)_{k \in \mathbb{N}}$ as follows:

$$
\left\{\begin{array}{l}
\left(y_{0}, f_{0}\right) \in \mathcal{A}, \\
\left(y_{k+1}, f_{k+1}\right)=\left(y_{k}, f_{k}\right)-\lambda_{k}\left(Y_{k}^{1}, F_{k}^{1}\right), \quad k \geq 0, \\
\lambda_{k}=\operatorname{argmin}_{\lambda \in[0, m]} E\left(\left(y_{k}, f_{k}\right)-\lambda\left(Y_{k}^{1}, F_{k}^{1}\right)\right)
\end{array}\right.
$$

where $\left(Y_{k}^{1}, F_{k}^{1}\right) \in \mathcal{A}_{0}$ is such that $F_{k}^{1}$ is a null control for $Y_{k}^{1}$, solution of

$$
\left\{\begin{array}{l}
\partial_{t} Y_{k}^{1}-\Delta Y_{k}^{1}+g^{\prime}\left(y_{k}\right) Y_{k}^{1}=F_{k}^{1} 1_{\omega}+\left(\partial_{t} y_{k}-\Delta y_{k}+g\left(y_{k}\right)-f_{k} 1_{\omega}\right) \text { in } Q_{T} \\
Y_{k}^{1}=0 \quad \text { on } \quad \Sigma_{T}, \quad Y_{k}^{1}(\cdot, 0)=0 \quad \text { in } \Omega
\end{array}\right.
$$

and minimizes the functional $J$ defined in Proposition 2.1. The direction $Y_{k}^{1}$ vanishes when $E$ vanishes. The real number $m \geq 1$ is arbitrarily fixed. It is used in the proof of convergence to bound the sequence of optimal descent steps $\lambda_{k}$.

We first perform the analysis assuming the non linear function $g$ in $W_{1}$, notably that $g^{\prime \prime} \in L^{\infty}(\mathbb{R})$ (the derivatives here are in the sense of distribution). We first prove the following lemma.

Lemma 3.6. Assume $g \in W_{1}$. Let $(y, f) \in \mathcal{A}$ and $\left(Y^{1}, F^{1}\right) \in \mathcal{A}_{0}$ defined by (3.3). For any $\lambda \in \mathbb{R}$ and $k \in \mathbb{N}$, the following estimate holds

$$
\sqrt{E\left((y, f)-\lambda\left(Y^{1}, F^{1}\right)\right)} \leq \sqrt{E(y, f)}\left(|1-\lambda|+\lambda^{2} C\left(s, \Omega, \omega, T,\left\|g^{\prime}\right\|_{\infty}\right)\left\|g^{\prime \prime}\right\|_{\infty} \sqrt{E(y, f)}\right)
$$

Proof. With $g \in W_{1}$, we write that

$$
\left|l\left(y,-\lambda Y^{1}\right)\right|=\left|g\left(y-\lambda Y^{1}\right)-g(y)+\lambda g^{\prime}(y) Y^{1}\right| \leq \frac{\lambda^{2}}{2}\left\|g^{\prime \prime}\right\|_{\infty}\left(Y^{1}\right)^{2}
$$

and obtain that

$$
\begin{aligned}
& 2 E\left((y, f)-\lambda\left(Y^{1}, F^{1}\right)\right) \\
& =\left\|\rho_{2}\left(\partial_{t} y-\Delta y+g(y)-f 1_{\omega}\right)-\lambda \rho_{2}\left(\partial_{t} Y^{1}-\Delta Y^{1}+g^{\prime}(y) Y^{1}-F 1_{\omega}\right)+\rho_{2} l\left(y,-\lambda Y^{1}\right)\right\|_{L^{2}\left(0, T ; H^{-1}(\Omega)\right)}^{2} \\
& =\left\|\rho_{2}(1-\lambda)\left(\partial_{t} y-\Delta y+g(y)-f 1_{\omega}\right)+\rho_{2} l\left(y,-\lambda Y^{1}\right)\right\|_{L^{2}\left(0, T ; H^{-1}(\Omega)\right)}^{2} \\
& \leq\left(\left\|\rho_{2}(1-\lambda)\left(\partial_{t} y-\Delta y+g(y)-f 1_{\omega}\right)\right\|_{L^{2}\left(0, T ; H^{-1}(\Omega)\right)}+\left\|\rho_{2} l\left(y,-\lambda Y^{1}\right)\right\|_{L^{2}\left(0, T ; H^{-1}(\Omega)\right)}\right)^{2} \\
& \leq 2\left(|1-\lambda| \sqrt{E(y, f)}+\frac{\lambda^{2}}{2 \sqrt{2}}\left\|g^{\prime \prime}\right\|_{\infty}\left\|\rho_{2}\left(Y^{1}\right)^{2}\right\|_{L^{2}\left(0, T ; H^{-1}(\Omega)\right)}\right)^{2} .
\end{aligned}
$$


For $d=3$ (similar estimates hold for $d=1$ and $d=2$ ), using the continuous embedding of $L^{6 / 5}(\Omega)$ into $H^{-1}(\Omega)$, we have:

$$
\begin{aligned}
\left\|\rho_{2}\left(Y^{1}\right)^{2}\right\|_{L^{2}\left(0, T ; H^{-1}(\Omega)\right)}^{2} & \leq C(\Omega)\left\|\rho_{2}\left(Y^{1}\right)^{2}\right\|_{L^{2}\left(0, T ; L^{6 / 5}(\Omega)\right)}^{2} \\
& \leq C(\Omega) \int_{0}^{T}\left\|\rho_{2} Y^{1}\right\|_{L^{3}(\Omega)}^{2}\left\|Y^{1}\right\|_{L^{2}(\Omega)}^{2} \\
& \leq C(\Omega) \int_{0}^{T}\left\|\rho Y^{1}\right\|_{L^{2}(\Omega)}\left\|\rho_{1} Y^{1}\right\|_{L^{6}(\Omega)}\left\|Y^{1}\right\|_{L^{2}(\Omega)}^{2} \\
& \leq C(\Omega) \int_{0}^{T}\left\|\rho Y^{1}\right\|_{L^{2}(\Omega)}\left\|\nabla\left(\rho_{1} Y^{1}\right)\right\|_{L^{2}(\Omega) d}\left\|Y^{1}\right\|_{L^{2}(\Omega)}^{2}
\end{aligned}
$$

From the definition of $\rho$ and $\rho_{1}$ we have $\nabla \rho_{1}=\frac{s \nabla \beta}{\ell(t)(T-t)} \rho_{1}=\frac{s \nabla \beta}{\ell(t)} \rho$ and therefore a.e. $t$ in $(0, T)$

$$
\begin{aligned}
\left\|\nabla\left(\rho_{1} Y^{1}\right)\right\|_{L^{2}(\Omega)^{d}} & \leq\left\|\nabla\left(\rho_{1}\right) Y^{1}\right\|_{L^{2}(\Omega)^{d}}+\left\|\rho_{1} \nabla Y^{1}\right\|_{L^{2}(\Omega)^{d}} \\
& \leq C\left(s, \Omega, \omega, T,\left\|g^{\prime}\right\|_{\infty}\right)\left\|\rho Y^{1}\right\|_{L^{2}(\Omega)}+\left\|\rho_{1} \nabla Y^{1}\right\|_{L^{2}(\Omega)^{d}}
\end{aligned}
$$

and thus

$$
\begin{aligned}
\left\|\rho_{2}\left(Y^{1}\right)^{2}\right\|_{L^{2}\left(0, T ; H^{-1}(\Omega)\right)}^{2} \leq & C\left(s, \Omega, \omega, T,\left\|g^{\prime}\right\|_{\infty}\right)\left\|\rho_{1} Y^{1}\right\|_{L^{\infty}\left(0, T ; L^{2}(\Omega)\right)}^{2}\left\|\rho Y^{1}\right\|_{L^{2}\left(Q_{T}\right)} \\
& \times\left(\left\|\rho Y^{1}\right\|_{L^{2}\left(Q_{T}\right)}+\left\|\rho_{1} \nabla Y^{1}\right\|_{L^{2}\left(Q_{T}\right)^{d}}\right)
\end{aligned}
$$

Using (3.5) and (3.6), we obtain

$$
\left\|\rho_{0}\left(Y^{1}\right)^{2}\right\|_{L^{2}\left(0, T ; H^{-1}(\Omega)\right)}^{2} \leq C\left(s, \Omega, \omega, T,\left\|g^{\prime}\right\|_{\infty}\right) E(y, f)^{2}
$$

from which we get (3.13).

Proceeding as in [23], we are now in position to prove the following convergence result for the sequence $\left(E\left(y_{k}, f_{k}\right)\right)_{(k \in \mathbb{N})}$.

Proposition 3.7. Assume $g \in W_{1}$. Let $\left(y_{k}, f_{k}\right)_{k \in \mathbb{N}}$ be the sequence defined by (3.11). Then $E\left(y_{k}, f_{k}\right) \rightarrow 0$ as $k \rightarrow \infty$. Moreover, there exists $k_{0} \in \mathbb{N}$ such that the sequence $\left(E\left(y_{k}, f_{k}\right)\right)_{k \geq k_{0}}$ decays quadratically.

Proof. We define the function $p_{k}$ as follows

$$
p_{k}(\lambda)=|1-\lambda|+\lambda^{2} c_{1} \sqrt{E\left(y_{k}, f_{k}\right)} \quad \text { with } \quad c_{1}:=C\left(s, \Omega, \omega, T,\left\|g^{\prime}\right\|_{\infty}\right)\left\|g^{\prime \prime}\right\|_{\infty}
$$

Lemma 3.6 with $(y, f)=\left(y_{k}, f_{k}\right)$ allows to write that

$$
c_{1} \sqrt{E\left(y_{k+1}, f_{k+1}\right)} \leq c_{1} \sqrt{E\left(y_{k}, f_{k}\right)} p_{k}\left(\widetilde{\lambda_{k}}\right), \quad \forall k \geq 0
$$

with $p_{k}\left(\widetilde{\lambda_{k}}\right):=\min _{\lambda \in[0, m]} p_{k}(\lambda)$.

If $c_{1} \sqrt{E\left(y_{0}, f_{0}\right)}<1$ (and thus $c_{1} \sqrt{E\left(y_{k}, f_{k}\right)}<1$ for all $k \in \mathbb{N}$ ) then

$$
p_{k}\left(\widetilde{\lambda_{k}}\right)=\min _{\lambda \in[0, m]} p_{k}(\lambda) \leq p_{k}(1)=c_{1} \sqrt{E\left(y_{k}, f_{k}\right)}
$$


and thus

$$
c_{1} \sqrt{E\left(y_{k+1}, f_{k+1}\right)} \leq\left(c_{1} \sqrt{E\left(y_{k}, f_{k}\right)}\right)^{2}
$$

implying that $c_{1} \sqrt{E\left(y_{k}, f_{k}\right)} \rightarrow 0$ as $k \rightarrow \infty$ with a quadratic rate.

If now $c_{1} \sqrt{E\left(y_{0}, f_{0}\right)} \geq 1$, we check that $I:=\left\{k \in \mathbb{N}, c_{1} \sqrt{E\left(y_{k}, f_{k}\right)} \geq 1\right\}$ is a finite subset of $\mathbb{N}$. For all $k \in I$, since $c_{1} \sqrt{E\left(y_{k}, f_{k}\right)} \geq 1$,

$$
\min _{\lambda \in[0, m]} p_{k}(\lambda)=\min _{\lambda \in[0,1]} p_{k}(\lambda)=p_{k}\left(\frac{1}{2 c_{1} \sqrt{E\left(y_{k}, f_{k}\right)}}\right)=1-\frac{1}{4 c_{1} \sqrt{E\left(y_{k}, f_{k}\right)}}
$$

and thus, for all $k \in I$,

$$
c_{1} \sqrt{E\left(y_{k+1}, f_{k+1}\right)} \leq\left(1-\frac{1}{4 c_{1} \sqrt{E\left(y_{k}, f_{k}\right)}}\right) c_{1} \sqrt{E\left(y_{k}, f_{k}\right)}=c_{1} \sqrt{E\left(y_{k}, f_{k}\right)}-\frac{1}{4} .
$$

This inequality implies that the sequence $\left(c_{1} \sqrt{E\left(y_{k}, f_{k}\right)}\right)_{k \in \mathbb{N}}$ strictly decreases and then that the sequence $\left(p_{k}\left(\widetilde{\lambda_{k}}\right)\right)_{k \in \mathbb{N}}$ decreases as well. Thus the sequence $\left(c_{1} \sqrt{E\left(y_{k}, f_{k}\right)}\right)_{k \in \mathbb{N}}$ decreases to 0 at least linearly and there exists $k_{0} \in \mathbb{N}$ such that for all $k \geq k_{0}, c_{1} \sqrt{E\left(y_{k}, f_{k}\right)}<1$, that is $I$ is a finite subset of $\mathbb{N}$. Arguing as in the first case, it follows that $c_{1} \sqrt{E\left(y_{k}, f_{k}\right)} \rightarrow 0$ as $k \rightarrow \infty$. In both cases, remark that $p_{k}\left(\widetilde{\lambda_{k}}\right)$ decreases with respect to $k$.

Remark 3.8. Writing from (3.19) that $c_{1} \sqrt{E\left(y_{k}, f_{k}\right)} \leq c_{1} \sqrt{E\left(y_{0}, f_{0}\right)}-\frac{k}{4}$ for all $k$ such that $c_{1} \sqrt{E\left(y_{k}, f_{k}\right)} \geq 1$, we obtain that

$$
k_{0} \leq\left\lfloor 4\left(c_{1} \sqrt{E\left(y_{0}, f_{0}\right)}-1\right)+1\right\rfloor
$$

where $\lfloor x\rfloor$ denotes the integer part of $x \in \mathbb{R}^{+}$.

We also have the following convergence of the optimal sequence $\left(\lambda_{k}\right)_{k \in \mathbb{N}}$.

Lemma 3.9. The sequence $\left(\lambda_{k}\right)_{k \in \mathbb{N}}$ defined in (3.11) converges to 1 as $k \rightarrow \infty$.

Proof. In view of (3.15), we have, as long as $E\left(y_{k}, f_{k}\right)>0$, since $\lambda_{k} \in[0, m]$

$$
\begin{aligned}
\left(1-\lambda_{k}\right)^{2}= & \frac{E\left(y_{k+1}, f_{k+1}\right)}{E\left(y_{k}, f_{k}\right)}-2\left(1-\lambda_{k}\right) \frac{\left\langle\rho_{2}\left(\partial_{t} y_{k}+\Delta y_{k}+g\left(y_{k}\right)-f_{k} 1_{\omega}\right), \rho_{2} l\left(y_{k}, \lambda_{k} Y_{k}^{1}\right)\right\rangle_{L^{2}\left(0, T ; H^{-1}(\Omega)\right)}}{E\left(y_{k}, f_{k}\right)} \\
& -\frac{\left\|\rho_{2} l\left(y_{k}, \lambda_{k} Y_{k}^{1}\right)\right\|_{L^{2}\left(0, T ; H^{-1}(\Omega)\right)}^{2}}{2 E\left(y_{k}\right)} \\
\leq & \frac{E\left(y_{k+1}, f_{k+1}\right)}{E\left(y_{k}, f_{k}\right)}-2\left(1-\lambda_{k}\right) \frac{\left\langle\rho_{2}\left(\partial_{t} y_{k}+\Delta y_{k}+g\left(y_{k}\right)-f_{k} 1_{\omega}\right), \rho_{2} l\left(y_{k}, \lambda_{k} Y_{k}^{1}\right)\right\rangle_{L^{2}\left(0, T ; H^{-1}(\Omega)\right)}}{E\left(y_{k}, f_{k}\right)} \\
\leq & \frac{E\left(y_{k+1}, f_{k+1}\right)}{E\left(y_{k}, f_{k}\right)}+2 \sqrt{2} m \frac{\sqrt{E\left(y_{k}, f_{k}\right)}\left\|\rho_{2} l\left(y_{k}, \lambda_{k} Y_{k}^{1}\right)\right\|_{L^{2}\left(0, T ; H^{-1}(\Omega)\right)}}{E\left(y_{k}, f_{k}\right)} \\
\leq & \frac{E\left(y_{k+1}, f_{k+1}\right)}{E\left(y_{k}, f_{k}\right)}+2 \sqrt{2} m \frac{\left\|\rho_{2} l\left(y_{k}, \lambda_{k} Y_{k}^{1}\right)\right\|_{L^{2}\left(0, T ; H^{-1}(\Omega)\right)}}{\sqrt{E\left(y_{k}, f_{k}\right)}} .
\end{aligned}
$$


But, from (3.14) and (3.16),

$$
\begin{aligned}
\left\|\rho_{2} l\left(y_{k}, \lambda_{k} Y_{k}^{1}\right)\right\|_{L^{2}\left(0, T ; H^{-1}(\Omega)\right)} & \leq \frac{\lambda_{k}^{2}}{2 \sqrt{2}}\left\|g^{\prime \prime}\right\|_{\infty}\left\|\rho_{2}\left(Y_{k}^{1}\right)^{2}\right\|_{L^{2}\left(0, T ; H^{-1}(\Omega)\right)} \\
& \leq m^{2}\left\|g^{\prime \prime}\right\|_{\infty} C\left(s, T, \Omega, \omega,\left\|g^{\prime}\right\|_{\infty}\right) E\left(y_{k}, f_{k}\right)
\end{aligned}
$$

and thus

$$
\left(1-\lambda_{k}\right)^{2} \leq \frac{E\left(y_{k+1}, f_{k+1}\right)}{E\left(y_{k}, f_{k}\right)}+m^{2}\left\|g^{\prime \prime}\right\|_{\infty} C\left(s, \Omega, \omega, T,\left\|g^{\prime}\right\|_{\infty}\right) \sqrt{E\left(y_{k}, f_{k}\right)} .
$$

Consequently, since $E\left(y_{k}, f_{k}\right) \rightarrow 0$ and $\frac{E\left(y_{k+1}, f_{k+1}\right)}{E\left(y_{k}, f_{k}\right)} \rightarrow 0$, we deduce that $\left(1-\lambda_{k}\right)^{2} \rightarrow 0$.

We are now in position to prove the following convergence result.

Theorem 3.10. Assume $g \in W_{1}$. Let $\left(y_{k}, f_{k}\right)_{k \in \mathbb{N}}$ be the sequence defined by (3.11). Then, $\left(y_{k}, f_{k}\right)_{k \in \mathbb{N}} \rightarrow(y, f)$ in $\mathcal{A}$ where $f$ is a null control for $y$ solution of (1.1). Moreover, the convergence is quadratic after a finite number of iterates.

Proof. For all $k \in \mathbb{N}$, let $F_{k}=-\sum_{n=0}^{k} \lambda_{n} F_{n}^{1}$ and $Y_{k}=\sum_{n=0}^{k} \lambda_{n} Y_{n}^{1}$. Let us prove that $\left(Y_{k}, F_{k}\right)_{k \in \mathbb{N}}$ converges in $\mathcal{A}_{0}$, i.e. that the series $\sum \lambda_{n}\left(F_{n}^{1}, Y_{n}^{1}\right)$ converges in $\mathcal{A}_{0}$. Using that $\left\|\left(Y_{k}^{1}, F_{k}^{1}\right)\right\|_{\mathcal{A}_{0}} \leq C \sqrt{E\left(y_{k}, f_{k}\right)}$ for all $k \in \mathbb{N}$ (see (3.4)), we write

$$
\sum_{n=0}^{k} \lambda_{n}\left\|\left(Y_{n}^{1}, F_{n}^{1}\right)\right\|_{\mathcal{A}_{0}} \leq m \sum_{n=0}^{k}\left\|\left(Y_{n}^{1}, F_{n}^{1}\right)\right\|_{\mathcal{A}_{0}} \leq C \sum_{n=0}^{k} \sqrt{E\left(y_{n}, f_{n}\right)} .
$$

$\operatorname{But}\left(\sqrt{E\left(y_{n}, f_{n}\right)}\right)_{k \in \mathbb{N}}$ and $\left(p_{k}\left(\widetilde{\lambda_{k}}\right)\right)_{k \in \mathbb{N}}$ are decreasing sequences so that

$$
\sqrt{E\left(y_{n}, f_{n}\right)} \leq p_{n}\left(\widetilde{\lambda_{n}}\right) \sqrt{E\left(y_{n-1}, f_{n-1}\right)} \leq p_{0}\left(\widetilde{\lambda_{0}}\right) \sqrt{E\left(y_{n-1}, f_{n-1}\right)} \leq p_{0}\left(\widetilde{\lambda_{0}}\right)^{n} \sqrt{E\left(y_{0}, f_{0}\right)} .
$$

Since $p_{0}(0)=1$ and $p_{0}^{\prime}(0)=-1$, we infer that $p_{0}\left(\widetilde{\lambda_{0}}\right)<1$ leading to

$$
\sum_{n=0}^{k} \sqrt{E\left(y_{n}, f_{n}\right)} \leq \sqrt{E\left(y_{0}, f_{0}\right)} \frac{1-p_{0}\left(\widetilde{\lambda_{0}}\right)^{k+1}}{1-p_{0}\left(\widetilde{\lambda_{0}}\right)} \leq \frac{\sqrt{E\left(y_{0}, f_{0}\right)}}{1-p_{0}\left(\widetilde{\lambda_{0}}\right)} .
$$

We deduce that the series $\sum_{n} \lambda_{n}\left(Y_{n}^{1}, F_{n}^{1}\right)$ is normally convergent and so convergent. Consequently, there exists $(Y, F) \in \mathcal{A}_{0}$ such that $\left(Y_{k}, F_{k}\right)_{k \in \mathbb{N}}$ converges to $(Y, F)$ in $\mathcal{A}_{0}$.

Denoting $y=y_{0}+Y$ and $f=f_{0}+F$, we then have that $\left(y_{k}, f_{k}\right)_{k \in \mathbb{N}}=\left(y_{0}+Y_{k}, f_{0}+F_{k}\right)_{k \in \mathbb{N}}$ converges to $(y, f)$ in $\mathcal{A}$.

It suffices now to verify that the limit $(y, f)$ satisfies $E(y, f)=0$. We write that $\left(Y_{k}^{1}, F_{k}^{1}\right) \in \mathcal{A}_{0}$ and $\left(y_{k}, f_{k}\right) \in \mathcal{A}$ solve

$$
\left\{\begin{array}{l}
\partial_{t} Y_{k}^{1}-\Delta Y_{k}^{1}+g^{\prime}\left(y_{k}\right) \cdot Y_{k}^{1}=F_{k}^{1} 1_{\omega}+\partial_{t} y_{k}-\Delta y_{k}+g\left(y_{k}\right)-f_{k} 1_{\omega} \quad \text { in } \quad Q_{T} \\
Y_{k}^{1}=0 \text { on } \Sigma_{T}, \quad Y_{k}^{1}(\cdot, 0)=0 \text { in } \Omega
\end{array}\right.
$$

Using that $\left(Y_{k}^{1}, F_{k}^{1}\right)$ goes to zero in $\mathcal{A}_{0}$ as $k \rightarrow \infty$, we pass to the limit in (3.20) and get, since $g \in W_{1}$, that $(y, f) \in \mathcal{A}$ solves (1.1), that is $E(y, f)=0$. 
In particular, along the sequence $\left(y_{k}, f_{k}\right)_{k \in \mathbb{N}}$ defined by (3.11), we have the following coercivity property for $E$, which confirms the strong convergence of the sequence $\left(y_{k}, f_{k}\right)_{k \in \mathbb{N}}$.

Proposition 3.11. Let $\left(y_{k}, f_{k}\right)_{k \in \mathbb{N}}$ defined by (3.11) and $(y, f)$ its limit. Then, there exists a positive constant $C=C\left(s, T, \Omega, \omega,\left\|g^{\prime}\right\|_{\infty}\right)$ such that

$$
\left\|(y, f)-\left(y_{k}, f_{k}\right)\right\|_{\mathcal{A}_{0}} \leq C \sqrt{E\left(y_{k}, f_{k}\right)}, \quad \forall k>0 .
$$

Proof. We write that

$$
\begin{aligned}
\left\|(y, f)-\left(y_{k}, f_{k}\right)\right\|_{\mathcal{A}_{0}} & =\left\|\sum_{p=k+1}^{\infty} \lambda_{p}\left(Y_{p}^{1}, F_{p}^{1}\right)\right\|_{\mathcal{A}_{0}} \leq m \sum_{p=k+1}^{\infty}\left\|\left(Y_{p}^{1}, F_{p}^{1}\right)\right\|_{\mathcal{A}_{0}} \\
& \leq m C \sum_{p=k+1}^{\infty} \sqrt{E\left(y_{p}, f_{p}\right)} \\
& \leq m C \sum_{p=k+1}^{\infty} p_{0}\left(\widetilde{\lambda}_{0}\right)^{p-k} \sqrt{E\left(y_{k}, f_{k}\right)} \\
& \leq m C \frac{p_{0}\left(\widetilde{\lambda}_{0}\right)}{1-p_{0}\left(\widetilde{\lambda}_{0}\right)} \sqrt{E\left(y_{k}, f_{k}\right)} .
\end{aligned}
$$

We emphasize, in view of the non uniqueness of the zeros of $E$, that an estimate (similar to (3.21)) of the form $\|(\bar{y}, \bar{f})-(y, f)\|_{\mathcal{A}_{0}} \leq C \sqrt{E(\bar{y}, \bar{f})}$ does not hold for all $(\bar{y}, \bar{f}) \in \mathcal{A}$. We also mention the fact that the sequence $\left(y_{k}, f_{k}\right)_{k>0}$ and its limits $(y, f)$ are uniquely determined from the initial guess $\left(y_{0}, f_{0}\right)$ and from our criterion of selection of the control $F^{1}$. In other words, the solution $(y, f)$ is unique up to the element $\left(y_{0}, f_{0}\right)$ and the functional $J$ defined in Proposition 2.1.

\subsection{The case $g \in W_{p}, 0 \leq p<1$ and additional remarks}

The results of the previous subsection devoted to the case $p=1$ still hold if we assume only that $g \in W_{p}$ for some $p \in(0,1)$. For any $g \in W_{p}$, we recall the notation $\left[g^{\prime}\right]_{p}:=\sup _{a, b \in \mathbb{R}, a \neq b} \frac{\left|g^{\prime}(a)-g^{\prime}(b)\right|}{|a-b|^{p}}$. We have the following result.

Theorem 3.12. Assume that there exists $p \in(0,1)$ such that $g \in W_{p}$. Let $\left(y_{k}, f_{k}\right)_{k \in \mathbb{N}}$ be the sequence defined by (3.11). Then, $\left(y_{k}, f_{k}\right)_{k \in \mathbb{N}} \rightarrow(y, f)$ in $\mathcal{A}$ where $f$ is a null control for $y$ solution of (1.1). Moreover, after a finite number of iterates, the rate of convergence is equal to $1+p$.

Proof. We briefly sketch the proof, close to the proof of Theorem 3.10 for the case $p=1$.

-We first prove for any $(y, f) \in \mathcal{A}$ and $\lambda \in \mathbb{R}$ the following inequality (similar to the inequality (3.13))

$$
E\left((y, f)-\lambda\left(Y^{1}, F^{1}\right)\right) \leq E(y, f)\left(|1-\lambda|+\lambda^{1+p} c_{1} E(y, f)^{p / 2}\right)^{2}
$$


with $c_{1}=C\left(s, T, \Omega, \omega,\left\|g^{\prime}\right\|_{\infty}\right)\left[g^{\prime}\right]_{p}$ and $\left(Y^{1}, F^{1}\right) \in \mathcal{A}_{0}$ the solution of $(3.12)$ which minimizes $J$. For any $(x, y) \in$ $\mathbb{R}^{2}$ and $\lambda \in \mathbb{R}$, we write $g(x+\lambda y)-g(x)=\int_{0}^{\lambda} y g^{\prime}(x+\xi y) \mathrm{d} \xi$ leading to

$$
\begin{aligned}
\left|g(x+\lambda y)-g(x)-\lambda g^{\prime}(x) y\right| & \leq \int_{0}^{\lambda}|y|\left|g^{\prime}(x+\xi y)-g^{\prime}(x)\right| \mathrm{d} \xi \\
& \leq \int_{0}^{\lambda}|y|^{1+p}|\xi|^{p} \frac{\left|g^{\prime}(x+\xi y)-g^{\prime}(x)\right|}{|\xi y|^{p}} \mathrm{~d} \xi \\
& \leq\left[g^{\prime}\right]_{s}|y|^{1+p} \frac{\lambda^{1+p}}{1+p} .
\end{aligned}
$$

It follows that

$$
\left|l\left(y,-\lambda Y^{1}\right)\right|=\left|g\left(y-\lambda Y^{1}\right)-g(y)+\lambda g^{\prime}(y) Y^{1}\right| \leq\left[g^{\prime}\right]_{p} \frac{\lambda^{1+p}}{1+p}\left|Y^{1}\right|^{1+p}
$$

and

$$
\begin{aligned}
\left\|\rho_{2} l\left(y, \lambda Y^{1}\right)\right\|_{L^{2}\left(0, T ; H^{-1}(\Omega)\right)} & \leq\left\|\rho_{2} l\left(y, \lambda Y^{1}\right)\right\|_{L^{2}\left(0, T ; L^{6 / 5}(\Omega)\right)} \\
& \leq\left[g^{\prime}\right]_{p} \frac{\lambda^{1+p}}{1+p}\left\|\rho_{2}\left|Y^{1}\right|^{1+p}\right\|_{L^{2}\left(0, T ; L^{6 / 5}(\Omega)\right)} .
\end{aligned}
$$

But

$$
\begin{aligned}
\left\|\rho_{2}\left|Y^{1}\right|^{1+p}\right\|_{L^{2}\left(0, T ; L^{6 / 5}(\Omega)\right)}^{2} & =\int_{0}^{T}\left\|\rho_{2}\left|Y^{1}\right|^{1+p}\right\|_{L^{6 / 5}(\Omega)}^{2} \leq \int_{0}^{T}\left\|\rho_{2} Y^{1}\right\|_{L^{3}(\Omega)}^{2}\left\|\left|Y^{1}\right|^{p}\right\|_{L^{2}(\Omega)}^{2} \\
& \leq \int_{0}^{T}\left\|\rho Y^{1}\right\|_{L^{2}(\Omega)}\left\|\rho_{1} Y^{1}\right\|_{L^{6}(\Omega)}\left\|Y^{1}\right\|_{L^{2 p}(\Omega)}^{2 p} \\
& \leq C(\Omega) \int_{0}^{T}\left\|\rho Y^{1}\right\|_{L^{2}(\Omega)}\left\|\nabla\left(\rho_{1} Y^{1}\right)\right\|_{L^{2}(\Omega)^{d}}\left\|Y^{1}\right\|_{L^{2 p}(\Omega)}^{2 p} \\
& \leq C(\Omega)\left\|\rho Y^{1}\right\|_{L^{2}\left(Q_{T}\right)}\left\|\nabla\left(\rho_{1} Y^{1}\right)\right\|_{L^{2}\left(Q_{T}\right)^{d}}\left\|Y^{1}\right\|_{L^{\infty}\left(0, T ; L^{2 s}(\Omega)\right)}^{2 p} \\
& \leq C(\Omega)\left\|\rho Y^{1}\right\|_{L^{2}\left(Q_{T}\right)}\left\|\nabla\left(\rho_{1} Y^{1}\right)\right\|_{L^{2}\left(Q_{T}\right)^{d}}\left\|Y^{1}\right\|_{L^{\infty}\left(0, T ; L^{2}(\Omega)\right)}^{2 p}
\end{aligned}
$$

Since $\left\|\nabla\left(\rho_{1} Y^{1}\right)\right\|_{L^{2}(\Omega)^{d}} \leq C\left(s, \Omega, \omega, T,\left\|g^{\prime}\right\|_{\infty}\right)\left\|\rho Y^{1}\right\|_{L^{2}(\Omega)}+\left\|\rho_{1} \nabla Y^{1}\right\|_{L^{2}(\Omega)^{d}}$, we finally get

$$
\begin{aligned}
\left\|\rho_{2}\left|Y^{1}\right|^{1+s}\right\|_{L^{2}\left(0, T ; L^{6 / 5}(\Omega)\right)}^{2} \leq & C\left(s, \Omega, \omega, T,\left\|g^{\prime}\right\|_{\infty}\right)\left\|\rho Y^{1}\right\|_{L^{2}\left(Q_{T}\right)} \\
& \times\left(\left\|\rho Y^{1}\right\|_{L^{2}\left(Q_{T}\right)}+\left\|\rho_{1} \nabla Y^{1}\right\|_{L^{2}\left(Q_{T}\right)^{d}}\right)\left\|\rho_{1} Y\right\|_{L^{\infty}\left(0, T ; L^{2}(\Omega)\right)}^{2 p}
\end{aligned}
$$

The first inequality of (3.15) then leads to (3.22).

- We then check that the sequence $\left(E\left(y_{k}, f_{k}\right)\right)_{k \in \mathbb{N}}$ goes to zero as $k \rightarrow \infty$. We define $p_{k}$ as follows

$$
p_{k}(\lambda)=|1-\lambda|+\lambda^{1+p} c_{1} E\left(y_{k}, f_{k}\right)^{p / 2}
$$

so that

$$
\sqrt{E\left(y_{k+1}, f_{k+1}\right)} \leq p_{k}\left(\widetilde{\lambda_{k}}\right) \sqrt{E\left(y_{k}, f_{k}\right)}, \quad \forall k \geq 0
$$


with $p_{k}\left(\widetilde{\lambda_{k}}\right)=\min _{\lambda \in[0, m]} p_{k}(\lambda)$. We have $p_{k}\left(\widetilde{\lambda}_{k}\right):=\min _{\lambda \in[0, m]} p_{k}(\lambda) \leq p_{k}(1)=c_{1} E\left(y_{k}, f_{k}\right)^{p / 2}$ and thus

$$
c_{2} \sqrt{E\left(y_{k+1}, f_{k+1}\right)} \leq\left(c_{2} \sqrt{E\left(y_{k}, f_{k}\right)}\right)^{1+p}, \quad c_{2}:=c_{1}^{1 / p} .
$$

If $c_{2} \sqrt{E\left(y_{0}, f_{0}\right)}<1$ (and thus $c_{2} \sqrt{E\left(y_{k}, f_{k}\right)}<1$ for all $k \in \mathbb{N}$ ) then the above inequality implies that $c_{2} \sqrt{E\left(y_{k}, f_{k}\right)} \rightarrow 0$ as $k \rightarrow \infty$. If $c_{2} \sqrt{E\left(y_{0}, f_{0}\right)} \geq 1$ then let $I=\left\{k \in \mathbb{N}, c_{2} \sqrt{E\left(y_{k}, f_{k}\right)} \geq 1\right\}$. $I$ is a finite subset of $\mathbb{N}$; for all $k \in I$, since $c_{2} \sqrt{E\left(y_{k}, f_{k}\right)} \geq 1$

$$
\min _{\lambda \in[0, m]} p_{k}(\lambda)=\min _{\lambda \in[0,1]} p_{k}(\lambda)=p_{k}\left(\frac{1}{(1+p)^{1 / p} c_{2} \sqrt{E\left(y_{k}, f_{k}\right)}}\right)=1-\frac{p}{(1+p)^{\frac{1}{p}+1}} \frac{1}{c_{2} \sqrt{E\left(y_{k}, f_{k}\right)}}
$$

and thus, for all $k \in I$,

$$
c_{2} \sqrt{E\left(y_{k+1}, f_{k+1}\right)} \leq\left(1-\frac{p}{(1+p)^{\frac{1}{p}+1}} \frac{1}{c_{2} \sqrt{E\left(y_{k}, f_{k}\right)}}\right) c_{2} \sqrt{E\left(y_{k}, f_{k}\right)}=c_{2} \sqrt{E\left(y_{k}, f_{k}\right)}-\frac{p}{(1+p)^{\frac{1}{p}+1}} .
$$

This inequality implies that the sequence $\left(c_{2} \sqrt{E\left(y_{k}, f_{k}\right)}\right)_{k \in \mathbb{N}}$ strictly decreases and then that the sequence $\left(p_{k}\left(\widetilde{\lambda_{k}}\right)\right)_{k \in \mathbb{N}}$ decreases as well. Thus the sequence $\left(c_{2} \sqrt{E\left(y_{k}, f_{k}\right)}\right)_{k \in \mathbb{N}}$ decreases to 0 at least linearly and there exists $k_{0} \in \mathbb{N}$ such that for all $k \geq k_{0}, c_{2} \sqrt{E\left(y_{k}, f_{k}\right)}<1$, that is $I$ is a finite subset of $\mathbb{N}$. Similarly, the optimal parameter $\lambda_{k}$ goes to one as $k \rightarrow \infty$.

- Using that the sequence $\left(E\left(y_{k}, f_{k}\right)\right)_{k \in \mathbb{N}}$ goes to zero, we conclude exactly as in the proof of Theorem 3.10.

On the other hand, if we assume only that $g$ belongs to $W_{0}$, then we can not expect the convergence of the sequence $\left(y_{k}, f_{k}\right)_{k>0}$ if $\left\|g^{\prime}\right\|_{\infty}$ is too large.

Remark 3.13. Assume that $g \in W_{0}$. Let any $(y, f) \in \mathcal{A}$ and $\left(Y^{1}, F^{1}\right)$ the solution of (3.3) which minimizes $J$. The following inequality holds:

$$
\sqrt{E\left((y, f)-\lambda\left(Y^{1}, F^{1}\right)\right)} \leq \sqrt{E(y, f)}\left(|1-\lambda|+\lambda C\left(s, \Omega, \omega, T,\left\|g^{\prime}\right\|_{\infty}\right)\left\|g^{\prime}\right\|_{\infty}\right)
$$

for all $\lambda \in \mathbb{R}$ where $C\left(s, \Omega, \omega, T,\left\|g^{\prime}\right\|_{\infty}\right) \geq 0$ increases with $\left\|g^{\prime}\right\|_{\infty}$. Indeed, this is a consequence of the following inequality, for all $(y, f) \in \mathcal{A},(Y, F) \in \mathcal{A}_{0}$ :

$$
\begin{aligned}
2 E\left((y, f)-\lambda\left(Y^{1}, F^{1}\right)\right) & \leq\left(\left\|\rho_{2}(1-\lambda)\left(\partial_{t} y-\Delta y+g(y)-f 1_{\omega}\right)\right\|_{L^{2}\left(0, T ; H^{-1}(\Omega)\right)}+\left\|\rho_{2} l\left(y, \lambda Y^{1}\right)\right\|_{L^{2}\left(0, T ; H^{-1}(\Omega)\right)}\right)^{2} \\
& \leq\left(|1-\lambda| \sqrt{2 E(y, f)}+2 \lambda\left\|(T-t)^{1 / 2} g^{\prime}(y)\right\|_{L^{\infty}\left(Q_{T}\right)}\|\rho Y\|_{L^{2}\left(Q_{T}\right)}\right)^{2} .
\end{aligned}
$$

Therefore, the sequence $\left(E\left(y_{k}, f_{k}\right)\right)_{k \in \mathbb{N}}$ decreases to 0 if $g$ satisfies

$$
C\left(s, \Omega, \omega, T,\left\|g^{\prime}\right\|_{\infty}\right)\left\|g^{\prime}\right\|_{\infty}<1
$$

Remark 3.14. The estimate (3.4) is a key point in the convergence analysis and is independent of the choice of the functional $J$ defined by $J\left(Y^{1}, F^{1}\right)=\frac{1}{2}\left\|\rho_{0} F^{1}\right\|_{L^{2}\left(q_{T}\right)}^{2}+\frac{1}{2}\|\rho Y\|_{L^{2}\left(Q_{T}\right)}^{2}$ (see Prop. 2.1) in order to select a pair $\left(Y^{1}, F^{1}\right)$ in $\mathcal{A}_{0}$. Thus, we may consider other weighted functionals, for instance $J\left(Y^{1}, F^{1}\right)=\frac{1}{2}\left\|\rho_{0} F^{1}\right\|_{L^{2}\left(q_{T}\right)}^{2}$ as discussed in [27]. 
Remark 3.15. If we introduce $F: \mathcal{A} \rightarrow L^{2}\left(0, T ; H^{-1}(\Omega)\right)$ by $F(y, f):=\rho_{2}\left(\partial_{t} y-\Delta y+g(y)-f 1_{\omega}\right)$, we get that $E(y, f)=\frac{1}{2}\|F(y, f)\|_{L^{2}\left(0, T ; H^{-1}(\Omega)\right)}^{2}$ and observe that, for $\lambda_{k}=1$, the algorithm (3.11) coincides with the Newton algorithm associated with the mapping $F$. This explains notably the quadratic convergence of Theorem 3.10 in the case $g \in W_{1}$ for which we have a control of $g^{\prime \prime}$ in $L^{\infty}\left(Q_{T}\right)$. The optimization of the parameter $\lambda_{k}$ allows to get a global convergence of the algorithm and leads to the so-called damped Newton method (for $F$ ). Under general hypothesis, global convergence for this kind of method is achieved, with a linear rate (for instance, we refer to [10], Thm. 8.7). As far as we know, the analysis of damped type Newton methods for partial differential equations has deserved very few attention in the literature. We mention $[22,30]$ in the context of fluid mechanics.

Remark 3.16. Suppose to simplify that $\lambda_{k}$ equals one (corresponding to the standard Newton method). Then, for each $k$, the optimal pair $\left(Y_{k}^{1}, F_{k}^{1}\right) \in \mathcal{A}_{0}$ is such that the element $\left(y_{k+1}, f_{k+1}\right)$ minimizes over $\mathcal{A}$ the functional $(z, v) \rightarrow J\left(z-y_{k}, v-f_{k}\right)$. Instead, we may also select the pair $\left(Y_{k}^{1}, F_{k}^{1}\right)$ such that the element $\left(y_{k+1}, f_{k+1}\right)$ minimizes the functional $(z, v) \rightarrow J(z, v)$. This leads to the following sequence $\left(y_{k}, f_{k}\right)_{k \in \mathbb{N}}$ in $\mathcal{A}$ defined by

$$
\left\{\begin{array}{l}
\partial_{t} y_{k+1}-\Delta y_{k+1}+g^{\prime}\left(y_{k}\right) y_{k+1}=f_{k+1} 1_{\omega}+g^{\prime}\left(y_{k}\right) y_{k}-g\left(y_{k}\right) \quad \text { in } Q_{T}, \\
y_{k}=0 \text { on } \Sigma_{T}, \quad\left(y_{k+1}(\cdot, 0), y_{k+1, t}(\cdot, 0)\right)=\left(u_{0}, u_{1}\right) \text { in } \Omega .
\end{array}\right.
$$

This is actually the formulation used in [13]. This formulation is different and the analysis of convergence (at least in the framework of our least-squares setting) is less direct because it is necessary to have a control of the right hand side term $g^{\prime}\left(y_{k}\right) y_{k}-g\left(y_{k}\right)$.

Remark 3.17. We emphasize that the explicit construction used here allows to recover the null controllability property of (1.1) for nonlinearities $g$ in $W_{p}$ for some $p \in(0,1]$. We do not use a fixed point argument as in [16]. On the other hand, the conditions we make on $g$ are more restrictives that in [16].

Eventually, it is also important to remark that these additional conditions does not imply a priori a contraction property of the operator $\Lambda$ introduced in [16]. If we consider the controlled pair associated with the weighted functional $J$, we can show as in [16] (where the control of minimal $L^{\infty}\left(Q_{T}\right)$ norm is used) that there exists a real $M$ large enough such that $\Lambda\left(B_{\infty}(0, M)\right) \subset B_{\infty}(0, M)$ (see [21]). Therefore, let $z_{i}$ in $L^{\infty}\left(Q_{T}\right)$, $i=1,2$ and assume that $\widetilde{g}^{\prime} \in L^{\infty}(0, M)$. If $\left(y_{z_{i}}, f_{z_{i}}\right)=\left(\Lambda\left(z_{i}\right), f_{z_{i}}\right), i=1,2$ are controlled pairs for the system (1.7) minimizing the functional $J$, then the following inequality holds:

$$
\left\|\rho\left(\Lambda\left(z_{1}\right)-\Lambda\left(z_{2}\right)\right)\right\|_{L^{2}\left(Q_{T}\right)} \leq C\left(s, \Omega, \omega, T,\|\tilde{g}\|_{L^{\infty}(0, M)}\right)\left\|\tilde{g}^{\prime}\right\|_{L^{\infty}(0, M)}\left\|u_{0}\right\|_{L^{2}(\Omega)}\left\|\rho\left(z_{1}-z_{2}\right)\right\|_{L^{2}\left(Q_{T}\right)}
$$

where $C\left(s, \Omega, \omega, T,\|\tilde{g}\|_{\infty}\right)$ is the constant appearing in (2.5). In view of Lemma 2.6 the pair $\left(y_{i}, f_{i}\right)=\left(\Lambda\left(z_{i}\right), f_{i}\right)$, $i=1,2$ satisfies $y_{i}=\rho^{-2} L_{\widetilde{g}\left(z_{i}\right)}^{\star} p_{i}$ and $f_{i}=-\left.\rho_{0}^{-2} p_{i}\right|_{q_{T}}$ for $p_{i} \in P_{\widetilde{g}\left(z_{i}\right)}$ solution of

$$
\left(p_{i}, q\right)_{P_{\widetilde{g}\left(z_{i}\right)}}=\int_{\Omega} u_{0} q(0), \quad \forall q \in P_{\widetilde{g}\left(z_{i}\right)} .
$$

i.e.

$$
\int_{Q_{T}} \rho^{-2} L_{\widetilde{g}\left(z_{i}\right)}^{\star} p_{i} L_{\widetilde{g}\left(z_{i}\right)}^{\star} q+\int_{q_{T}} \rho_{0}^{-2} p_{i} q=\int_{\Omega} u_{0} q(0), \quad \forall q \in P_{\widetilde{g}\left(z_{i}\right)}
$$

leading to

$$
\begin{aligned}
& \int_{Q_{T}} \rho^{-2} L_{\widetilde{g}\left(z_{2}\right)}^{\star}\left(p_{2}-p_{1}\right) L_{\widetilde{g}\left(z_{2}\right)}^{\star} q+\int_{q_{T}} \rho_{0}^{-2}\left(p_{2}-p_{1}\right) q \\
& =-\int_{Q_{T}} \rho^{-2}\left(\left(L_{\widetilde{g}\left(z_{2}\right)}^{\star}-L_{\widetilde{g}\left(z_{1}\right)}^{\star}\right) p_{1} L_{\widetilde{g}\left(z_{2}\right)}^{\star} q+L_{\widetilde{g}\left(z_{1}\right)}^{\star} p_{1}\left(L_{\widetilde{g}\left(z_{2}\right)}^{\star}-L_{\widetilde{g}\left(z_{1}\right)}^{\star}\right) q\right) \quad \forall q \in P_{\widetilde{g}\left(z_{i}\right)} .
\end{aligned}
$$


Using that $\left(L_{\widetilde{g}\left(z_{2}\right)}^{\star}-L_{\widetilde{g}\left(z_{1}\right)}^{\star}\right) q=\left(\widetilde{g}\left(z_{2}\right)-\widetilde{g}\left(z_{1}\right)\right) q$ for all $q \in P_{\widetilde{g}\left(z_{i}\right)}$ and taking $q=p_{2}-p_{1}$, we obtain

$$
\begin{aligned}
&\left\|p_{2}-p_{1}\right\|_{P_{\widetilde{g}\left(z_{2}\right)}}^{2}=\left\|\rho^{-1} L_{\widetilde{g}\left(z_{2}\right)}^{\star}\left(p_{2}-p_{1}\right)\right\|_{L^{2}\left(Q_{T}\right)}^{2}+\left\|\rho_{0}^{-1}\left(p_{2}-p_{1}\right)\right\|_{L^{2}\left(q_{T}\right)}^{2} \\
& \leq\left\|\rho^{-1}\left(\widetilde{g}\left(z_{2}\right)-\widetilde{g}\left(z_{1}\right)\right) p_{1}\right\|_{L^{2}\left(Q_{T}\right)}\left\|\rho^{-1} L_{\widetilde{g}\left(z_{2}\right)}^{\star}\left(p_{2}-p_{1}\right)\right\|_{L^{2}\left(Q_{T}\right)} \\
& \quad+\left\|\rho^{-1} L_{\widetilde{g}\left(z_{1}\right)}^{\star} p_{1}\right\|_{L^{2}\left(Q_{T}\right)}\left\|\rho^{-1}\left(\widetilde{g}\left(z_{2}\right)-\widetilde{g}\left(z_{1}\right)\right)\left(p_{2}-p_{1}\right)\right\|_{L^{2}\left(Q_{T}\right)} \\
& \leq\left\|\rho^{-1}\left(\widetilde{g}\left(z_{2}\right)-\widetilde{g}\left(z_{1}\right)\right) p_{1}\right\|_{L^{2}\left(Q_{T}\right)}\left\|p_{2}-p_{1}\right\|_{P_{\widetilde{g}\left(z_{2}\right)}}+\left\|\rho \Lambda\left(z_{1}\right)\right\|_{L^{2}\left(Q_{T}\right)}\left\|\rho^{-1}\left(\widetilde{g}\left(z_{2}\right)-\widetilde{g}\left(z_{1}\right)\right)\left(p_{2}-p_{1}\right)\right\|_{L^{2}\left(Q_{T}\right)} .
\end{aligned}
$$

Moreover, from Lemma 3.2 of [13], there exists $C>0$ such that $\left\|(\xi \rho)^{-1} q\right\|_{L^{\infty}\left(Q_{T}\right)} \leq C\|q\|_{P_{\widetilde{g}\left(z_{i}\right)}}$ for all $q \in P_{\widetilde{g}\left(z_{i}\right)}$ and $\xi=(T-t)^{-1 / 2}$. It follows that

$$
\begin{aligned}
\left\|\rho^{-1}\left(\widetilde{g}\left(z_{2}\right)-\widetilde{g}\left(z_{1}\right)\right) p_{1}\right\|_{L^{2}\left(Q_{T}\right)} & \leq\left\|\rho\left(\widetilde{g}\left(z_{2}\right)-\widetilde{g}\left(z_{1}\right)\right)\right\|_{L^{2}\left(Q_{T}\right)}\left\|\rho^{-2} p_{1}\right\|_{L^{\infty}\left(Q_{T}\right)} \\
& \leq\left\|\widetilde{g}^{\prime}\right\|_{L^{\infty}(0, M)}\left\|\rho\left(z_{1}-z_{2}\right)\right\|_{L^{2}\left(Q_{T}\right)}\left\|\zeta \rho^{-1}\right\|_{L^{\infty}\left(Q_{T}\right)}\left\|(\zeta \rho)^{-1} p_{1}\right\|_{L^{\infty}\left(Q_{T}\right)} \\
& \leq C\left\|\widetilde{g}^{\prime}\right\|_{L^{\infty}(0, M)}\left\|\zeta \rho^{-1}\right\|_{L^{\infty}\left(Q_{T}\right)}\left\|p_{1}\right\|_{P_{\widetilde{g}\left(z_{i}\right)}}\left\|\rho\left(z_{1}-z_{2}\right)\right\|_{L^{2}\left(Q_{T}\right)}
\end{aligned}
$$

and

$$
\begin{aligned}
\left\|\rho^{-1}\left(\widetilde{g}\left(z_{2}\right)-\widetilde{g}\left(z_{1}\right)\right)\left(p_{2}-p_{1}\right)\right\|_{L^{2}\left(Q_{T}\right)} & \leq\left\|\widetilde{g}^{\prime}\right\|_{L^{\infty}(0, M)}\left\|\rho\left(z_{1}-z_{2}\right)\right\|_{L^{2}\left(Q_{T}\right)}\left\|\zeta \rho^{-1}\right\|_{L^{\infty}\left(Q_{T}\right)}\left\|(\zeta \rho)^{-1}\left(p_{2}-p_{1}\right)\right\|_{L^{\infty}\left(Q_{T}\right)} \\
& \leq C\left\|\widetilde{g}^{\prime}\right\|_{L^{\infty}(0, M)}\left\|\zeta \rho^{-1}\right\|_{L^{\infty}\left(Q_{T}\right)}\left\|p_{2}-p_{1}\right\|_{P_{\tilde{g}\left(z_{2}\right)}}\left\|\rho\left(z_{1}-z_{2}\right)\right\|_{L^{2}\left(Q_{T}\right)} .
\end{aligned}
$$

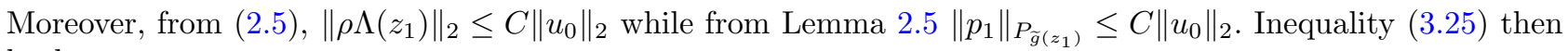
leads to

$$
\left\|\rho^{-1} L_{\widetilde{g}\left(z_{2}\right)}^{\star}\left(p_{2}-p_{1}\right)\right\|_{L^{2}\left(Q_{T}\right)} \leq C\left\|\widetilde{g}^{\prime}\right\|_{L_{l o c}^{\infty}}\left\|\zeta \rho^{-1}\right\|_{L^{\infty}\left(Q_{T}\right)}\left\|\rho\left(z_{1}-z_{2}\right)\right\|_{L^{2}\left(Q_{T}\right)}\left\|u_{0}\right\|_{L^{2}(\Omega)}
$$

Eventually,

$$
\begin{aligned}
\left\|\rho\left(\Lambda\left(z_{2}\right)-\Lambda\left(z_{1}\right)\right)\right\|_{2} & =\left\|\rho^{-1} L_{\widetilde{\widetilde{g}}\left(z_{2}\right)}^{\star}\left(p_{2}\right)-\rho^{-1} L_{\widetilde{\widetilde{g}}\left(z_{1}\right)}^{\star}\left(p_{1}\right)\right\|_{L^{2}\left(Q_{T}\right)} \\
& =\left\|\rho^{-1} L_{\widetilde{g}\left(z_{2}\right)}^{\star}\left(p_{2}-p_{1}\right)-\rho^{-1}\left(L_{\widetilde{g}\left(z_{1}\right)}^{\star}-L_{\widetilde{g}\left(z_{2}\right)}^{\star}\right)\left(p_{1}\right)\right\|_{L^{2}\left(Q_{T}\right)} \\
& =\left\|\rho^{-1} L_{\widetilde{\widetilde{g}}\left(z_{2}\right)}^{\star}\left(p_{2}-p_{1}\right)-\rho^{-1}\left(\widetilde{g}\left(z_{1}\right)-\widetilde{g}\left(z_{2}\right)\right)\left(p_{1}\right)\right\|_{L^{2}\left(Q_{T}\right)} \\
& \leq\left\|\rho^{-1} L_{\widetilde{g}\left(z_{2}\right)}^{\star}\left(p_{2}-p_{1}\right)\right\|_{L^{2}\left(Q_{T}\right)}+\left\|\rho^{-1}\left(\widetilde{g}\left(z_{1}\right)-\widetilde{g}\left(z_{2}\right)\right)\left(p_{1}\right)\right\|_{L^{2}\left(Q_{T}\right)}
\end{aligned}
$$

Combining (3.26) and (3.27), we finally get the estimate (3.24). It follows that a smallness assumption on $\left\|\widetilde{g}^{\prime}\right\|_{L^{\infty}(0, M)}$ leads to a contraction property for $\Lambda$. We observe that $\widetilde{g}^{\prime}(r)=\left(g^{\prime}(r) r-g(r)\right) / r^{2}, r \neq 0$ so that $\widetilde{g}^{\prime}$ belongs to $L^{\infty}(0, M)$ if $g^{\prime \prime}$ belongs to $L^{\infty}(0, M)$.

\section{NumericAl ILLUSTRATIONS}

We illustrate in this section our results of convergence. We provide some practical details of the algorithm (3.11) then discuss some experiments in the one dimensional case. Approximations of null controls for the heat equation is a delicate issue: we mention the seminal work [5] dealing with the control of minimal $L^{2}$-norm which is very oscillatory near the final time $t=T$ and therefore difficult to construct. We also mention [19, 29] where this is discussed at length. On the other hand, introduction of very specific weights in the cost functional $J$ allows to avoid oscillatory phenomena and leads to a robust method and strong convergent approximations with respect to the discretization parameter. We refer to $[14,15]$. 


\subsection{Approximation - Algorithm}

Each iterate of the algorithm (3.11) requires the determination of the null control of $F_{k}^{1}$ for $Y_{k}^{1}$ solution of

$$
\left\{\begin{array}{l}
\partial_{t} Y_{k}^{1}-\Delta Y_{k}^{1}+g^{\prime}\left(y_{k}\right) Y_{k}^{1}=F_{k}^{1} 1_{\omega}+B_{k} \quad \text { in } Q_{T}, \\
Y_{k}^{1}=0 \quad \text { on } \quad \Sigma_{T}, \quad Y_{k}^{1}(\cdot, 0)=0 \quad \text { in } \Omega
\end{array}\right.
$$

with $B_{k}:=\partial_{t} y_{k}-\Delta y_{k}+g\left(y_{k}\right)-f_{k} 1_{\omega}$. From Lemma 2.6, the pair $\left(F_{k}^{1}, Y_{k}^{1}\right)$ which minimizes the functional $J$ is given by $Y_{k}^{1}=\rho^{-2} L_{g^{\prime}\left(y_{k}\right)}^{\star} p_{k}$ and $F_{k}^{1}=-\rho_{0}^{-2} p_{k} 1_{q_{T}}$ where $p_{k} \in P$ solves the formulation

$$
\int_{Q_{T}} \rho^{-1} L_{g^{\prime}\left(y_{k}\right)}^{\star} p_{k} \rho^{-1} L_{g^{\prime}\left(y_{k}\right)}^{\star} \bar{p}+\int_{q_{T}} \rho_{0}^{-1} p_{k} \rho_{0}^{-1} \bar{p}=\int_{0}^{T}<\rho_{2} B_{k}, \rho_{2}^{-1} \bar{p}>_{H^{-1}(\Omega), H_{0}^{1}(\Omega)} \mathrm{d} t \quad \forall \bar{p} \in P .
$$

The numerical approximation of this variational formulation (of second order in time and fourth order in space) has been discussed at length in [14]. In order, first to avoid numerical instabilities (due to the presence of exponential functions in the formulation), and second to make appear explicitly the controlled solution, we introduce the variables $m_{k}:=\rho_{0}^{-1} p_{k}, z_{k}:=\rho^{-1} L_{g^{\prime}\left(y_{k}\right)}^{\star} p_{k}$. Since $\rho_{2}^{-1} p \in L^{2}\left(0, T ; H_{0}^{1}(\Omega)\right)$, we obtain notably that $\rho_{2}^{-1} p=\rho_{2}^{-1} \rho_{0} m=(T-t) m \in L^{2}\left(0, T ; H_{0}^{1}(\Omega)\right)$. From (4.2), the pair $\left(m_{k}, z_{k}\right) \in \mathcal{M} \times L^{2}\left(Q_{T}\right)$ with $\mathcal{M}:=\rho_{0}^{-1} P$ solves

$$
\int_{Q_{T}} z_{k} \bar{z}+\int_{q_{T}} m_{k} \bar{m}=\int_{0}^{T}<\rho_{2} B_{k},(T-t) \bar{m}>_{H^{-1}(\Omega), H_{0}^{1}(\Omega)} \mathrm{d} t \quad \forall(\bar{m}, \bar{z}) \in \mathcal{M} \times L^{2}\left(Q_{T}\right)
$$

subjected to the constraint $z_{k}=\rho^{-1} L_{g^{\prime}\left(y_{k}\right)}^{\star}\left(\rho_{0} m_{k}\right)$. This constraint leads to the following well-posed mixed formulation: find $\left(m_{k}, z_{k}, \eta_{k}\right) \in \mathcal{M} \times L^{2}\left(Q_{T}\right) \times L^{2}\left(Q_{T}\right)$ solution of

$$
\left\{\begin{array}{c}
\int_{Q_{T}} z_{k} \bar{z}+\int_{q_{T}} m_{k} \bar{m}+\int_{Q_{T}} \eta_{k}\left(\bar{z}-\rho^{-1} L_{g^{\prime}(y)}^{\star}\left(\rho_{0} \bar{m}\right)\right) \\
=\int_{0}^{T}<\rho_{2} B_{k},(T-t) \bar{m}>_{H^{-1}(\Omega), H_{0}^{1}(\Omega)} \mathrm{d} t, \quad \forall(\bar{m}, \bar{z}) \in \mathcal{M} \times L^{2}\left(Q_{T}\right), \\
\int_{Q_{T}} \bar{\eta}\left(z_{k}-\rho^{-1} L_{g^{\prime}\left(y_{k}\right)}^{\star}\left(\rho_{0} m\right)\right)=0, \quad \forall \bar{\eta} \in L^{2}\left(Q_{T}\right) .
\end{array}\right.
$$

The variable $\eta_{k} \in L^{2}\left(Q_{T}\right)$ acts as a Lagrange multiplier. Moreover, from the unique solution $\left(m_{k}, z_{k}\right)$, we get the explicit form of the controlled pair $\left(Y_{k}^{1}, F_{k}^{1}\right)$ as follows: $Y_{k}^{1}=\rho^{-1} z_{k}, F_{k}^{1}=-\rho_{0}^{-1} m_{k} 1_{q_{T}}$.

The algorithm associated with the sequence $\left(y_{k}, f_{k}\right)_{k \in \mathbb{N}}$ (see (3.11)) may be developed as follows: given $\epsilon>0$ and $m \geq 1$,

1. We determine the controlled pair $\left(y_{0}, f_{0}\right) \in \mathcal{A}$ which minimizes the functional $J$ associated with the linear case (for which $g \equiv 0$ in $(1.1))$. $\left(y_{0}, f_{0}\right)$ is given by $\left(y_{0}, f_{0}\right)=\left(\rho^{-1} z_{0},-\rho_{0}^{-1} m_{0} 1_{q_{T}}\right)$ where $\left(m_{0}, z_{0}\right) \in$ $\mathcal{M} \times L^{2}\left(Q_{T}\right)$ together with $\eta \in L^{2}\left(Q_{T}\right)$ solve the formulation:

$$
\left\{\begin{array}{l}
\int_{Q_{T}} z \bar{z}+\int_{q_{T}} m \bar{m}+\int_{Q_{T}} \eta\left(\bar{z}-\rho^{-1} L_{0}^{\star}\left(\rho_{0} \bar{m}\right)\right)=\int_{\Omega} \rho_{0}(\cdot, 0) u_{0} \bar{m}(\cdot, 0), \quad \forall(\bar{m}, \bar{z}) \in \mathcal{M} \times L^{2}\left(Q_{T}\right), \\
\int_{Q_{T}} \bar{\eta}\left(z-\rho^{-1} L_{0}^{\star}\left(\rho_{0} m\right)\right)=0, \quad \forall \bar{\eta} \in L^{2}\left(Q_{T}\right) .
\end{array}\right.
$$

In view of Proposition 2.1, we check that $\left(y_{0}, f_{0}\right)$ belongs to $\mathcal{A}$. 
2. Assume now that $\left(y_{k}, f_{k}\right)$ is computed for some $k \geq 0$. We then compute $c_{k} \in L^{2}\left(0, T ; H_{0}^{1}(\Omega)\right)$, unique solution of

$$
\int_{Q_{T}} \nabla c_{k} \cdot \nabla \bar{c}=\int_{0}^{T}<\rho_{2}\left(\partial_{t} y_{k}-\Delta y_{k}+g\left(y_{k}\right)-f_{k} 1_{\omega}\right), \bar{c}>_{H^{-1}(\Omega), H_{0}^{1}(\Omega)}, \quad \forall \bar{c} \in L^{2}\left(0, T ; H_{0}^{1}(\Omega)\right)
$$

and then $E\left(y_{k}, f_{k}\right)=\frac{1}{2}\left\|\rho_{2}\left(\partial_{t} y_{k}-\Delta y_{k}+g\left(y_{k}\right)-f_{k} 1_{\omega}\right)\right\|_{L^{2}\left(0, T ; H^{-1}(\Omega)\right)}^{2}=\frac{1}{2}\left\|\nabla c_{k}\right\|_{L^{2}\left(Q_{T}\right)}^{2}$.

3. If $E\left(y_{k}, f_{k}\right)<\epsilon$, the approximate controlled pair is given by $(\bar{y}, \bar{f})=\left(y_{k}, f_{k}\right)$ and the algorithm stops. Otherwise, we determine the solution $\left(Y_{k}^{1}, F_{k}^{1}\right)=\left(\rho^{-1} z_{k},-\rho_{0}^{-1} m_{k} 1_{q_{T}}\right)$ where $\left(m_{k}, z_{k}\right)$ together with $\eta_{k} \in$ $L^{2}\left(Q_{T}\right)$ solve $(4.3)$.

4. Set $\left(y_{k+1}, f_{k+1}\right)=\left(y_{k}, f_{k}\right)-\lambda_{k}\left(Y_{k}^{1}, F_{k}^{1}\right)$ where $\lambda_{k}$ minimizes over $[0, m]$ the scalar functional $\lambda \rightarrow$ $E\left(\left(y_{k}, f_{k}\right)-\lambda\left(Y_{k}^{1}, F_{k}^{1}\right)\right)$ defined by (see (3.15))

$$
E\left(\left(y_{k}, f_{k}\right)-\lambda\left(Y_{k}^{1}, F_{k}^{1}\right)\right)=\frac{1}{2}\left\|\rho_{2}(1-\lambda)\left(\partial_{t} y_{k}-\Delta y_{k}+g\left(y_{k}\right)-f_{k} 1_{\omega}\right)+\rho_{2} l\left(y_{k},-\lambda Y_{k}^{1}\right)\right\|_{L^{2}\left(0, T ; H^{-1}(\Omega)\right)}^{2}
$$

with $l\left(y_{k},-\lambda Y_{k}^{1}\right)=g\left(y_{k}-\lambda Y_{k}^{1}\right)-g\left(y_{k}\right)+\lambda g^{\prime}\left(y_{k}\right) Y_{k}^{1}$. The minimization is performed using a line search method. Return to step 2.

We use the conformal space-time finite element method described in [14]. We consider a regular family $\mathcal{T}=$ $\left\{\mathcal{T}_{h} ; h>0\right\}$ of triangulation of $Q_{T}$ such that $\overline{Q_{T}}=\cup_{K \in \mathcal{T}_{h}} K$. The family $\mathcal{T}$ is indexed by $h=\max _{K \in \mathcal{T}_{h}} \operatorname{diam}(K)$. The variable $z_{k}$ and $\eta_{k}$ are approximated with the space $P_{h}=\left\{p_{h} \in C\left(\overline{Q_{T}}\right) ;\left.p_{h}\right|_{K} \in \mathbb{P}_{1}(K), \forall K \in \mathcal{T}_{h}\right\} \subset L^{2}\left(Q_{T}\right)$ where $\mathbb{P}_{1}(K)$ denotes the space of affine functions both in $x$ and $t$. The variable $m_{k}$ is approximated with the space $V_{h}=\left\{v_{h} \in C^{1}\left(\overline{Q_{T}}\right) ;\left.v_{h}\right|_{K} \in \mathbb{P}(K), \forall K \in \mathcal{T}_{h}\right\} \subset \mathcal{M}$ where $\mathbb{P}(K)$ denotes the Hsieh-Clough-Tocher $C^{1}$ element (we refer to [7], p. 356). These conformal approximation leads to a strong convergent approximation of the controlled pairs $\left(y_{0}, f_{0}\right),\left(Y_{k}^{1}, F_{k}^{1}\right)$ (and then $\left.\left(y_{k}, f_{k}\right)\right)$ for every $k \in \mathbb{N}$ with respect to the parameter $h$ for the norm associated with $P$ : precisely

$$
\left\|\rho_{0}\left(f_{k, h}-f_{k}\right)\right\|_{L^{2}\left(q_{T}\right)}+\left\|\rho\left(y_{k, h}-y_{k}\right)\right\|_{L^{2}\left(Q_{T}\right)} \rightarrow 0 \text { as } h \rightarrow 0^{+}, \quad \forall k \in \mathbb{N}
$$

Remark that this strong convergent approximation of the linear control $f_{k}$ together with the convergent result of Theorem 3.10 allows to get, for $k$ large enough and $h$ small enough, an approximation $f_{k, h}$ of a control $f$ for the nonlinear equation (1.1), writing that

$$
\left\|\rho_{0}\left(f-f_{k, h}\right)\right\|_{L^{2}\left(q_{T}\right)} \leq\left\|\rho_{0}\left(f-f_{k}\right)\right\|_{L^{2}\left(q_{T}\right)}+\left\|\rho_{0}\left(f_{k}-f_{k, h}\right)\right\|_{L^{2}\left(q_{T}\right)}, \quad \forall k \in \mathbb{N}, \forall h>0 .
$$

\subsection{Experiments}

We present some numerical experiments in the one dimensional setting and $\Omega=(0,1)$. The control is located on $\omega=(0.1,0.3)$. We consider $T=1 / 2$; moreover, in order to reduce the dissipation of the solution of (1.1) when $g \equiv 0$, we replace the term $-\Delta y$ in (1.1) by $-\nu \Delta y$ with $\nu=10^{-1}$. We consider the nonlinear even function $g$ as follows

$$
g(r)=\left\{\begin{array}{lr}
l(r), & r \in[-a, a] \\
-|r|^{\alpha} \ln ^{3 / 2}(1+|r|), & |r| \geq a
\end{array}\right.
$$

with $a, \alpha \in(0,1) . l$ denotes the (even) polynomial of order two so that $g(0)=0$ and $g \in C^{1}([-a, a])$ : this requires $l(0)=0, l(a)=-|a|^{\alpha} \ln ^{3 / 2}(1+|a|)$ and $\left(-|r|^{\alpha} \ln ^{3 / 2}(1+|r|)\right)^{\prime}(r=a)=l^{\prime}(a)$. We use in the sequel the values 
TABLE 1. Results for the algorithm (3.11); $\beta=10$.

\begin{tabular}{ccccccc}
\hline \multirow{2}{*}{$\sharp$ iteration $k$} & $\frac{\left\|y_{k}-y_{k-1}\right\|_{L^{2}\left(Q_{T}\right)}}{\left\|y_{k-1}\right\|_{L^{2}\left(Q_{T}\right)}}$ & $\frac{\left\|f_{k}-f_{k-1}\right\|_{L^{2}\left(q_{T}\right)}}{\left\|f_{k-1}\right\|_{L^{2}\left(q_{T}\right)}}$ & $\left\|y_{k}\right\|_{L^{2}\left(Q_{T}\right)}$ & $\left\|f_{k}\right\|_{L^{2}\left(q_{T}\right)}$ & $\sqrt{2 E\left(y_{k}, f_{k}\right)}$ & $\lambda_{k}$ \\
\hline 0 & - & - & 4.528 & 4.391 & $5.58 \times 10^{-1}$ & 0.961 \\
1 & $1.83 \times 10^{-2}$ & $1.28 \times 10^{-3}$ & 4.651 & 4.402 & $1.81 \times 10^{-3}$ & 0.996 \\
2 & $4.45 \times 10^{-4}$ & $9.07 \times 10^{-5}$ & 4.661 & 4.403 & $2.72 \times 10^{-6}$ & 1. \\
3 & $1.12 \times 10^{-6}$ & $3.74 \times 10^{-7}$ & 4.662 & 4.404 & $4.88 \times 10^{-8}$ & - \\
\hline
\end{tabular}

TABLE 2. Results for the algorithm $(3.11) ; \beta=10^{2}$.

\begin{tabular}{|c|c|c|c|c|c|c|}
\hline$\sharp$ iteration $k$ & $\frac{\left\|y_{k}-y_{k-1}\right\|_{L^{2}\left(Q_{T}\right)}}{\left\|y_{k-1}\right\|_{L^{2}\left(Q_{T}\right)}}$ & $\frac{\left\|f_{k}-f_{k-1}\right\|_{L^{2}\left(q_{T}\right)}}{\left\|f_{k-1}\right\|_{L^{2}\left(q_{T}\right)}}$ & $\left\|y_{k}\right\|_{L^{2}\left(Q_{T}\right)}$ & $\left\|f_{k}\right\|_{L^{2}\left(q_{T}\right)}$ & $\sqrt{2 E\left(y_{k}, f_{k}\right)}$ & $\lambda_{k}$ \\
\hline 0 & - & - & 45.28 & 43.91 & $9.31 \times 10^{-1}$ & 0.534 \\
\hline 1 & $8.41 \times 10^{-1}$ & $1.23 \times 10^{-2}$ & 35.8908 & 38.76 & $1.12 \times 10^{-1}$ & 0.591 \\
\hline 2 & $1.93 \times 10^{-1}$ & $2.91 \times 10^{-3}$ & 36.7302 & 38.92 & $3.40 \times 10^{-2}$ & 0.701 \\
\hline 3 & $3.65 \times 10^{-2}$ & $1.01 \times 10^{-3}$ & 37.0919 & 39.12 & $6.12 \times 10^{-3}$ & 0.812 \\
\hline 4 & $1.12 \times 10^{-2}$ & $2.69 \times 10^{-4}$ & 37.2124 & 40.01 & $1.12 \times 10^{-3}$ & 0.881 \\
\hline 5 & $3.23 \times 10^{-4}$ & $4.23 \times 10^{-5}$ & 37.2426 & 40.04 & $2.13 \times 10^{-4}$ & 0.912 \\
\hline 6 & $1.27 \times 10^{-5}$ & $6.23 \times 10^{-6}$ & 37.2518 & 40.05 & $3.05 \times 10^{-5}$ & 0.999 \\
\hline 7 & $5.09 \times 10^{-6}$ & $8.12 \times 10^{-7}$ & 37.2520 & 40.05 & $2.10 \times 10^{-6}$ & 0.999 \\
\hline 8 & $7.40 \times 10^{-8}$ & $8.21 \times 10^{-9}$ & 37.2520 & 40.05 & $5.10 \times 10^{-9}$ & - \\
\hline
\end{tabular}

$a=10^{-1}$ and $\alpha=0.95$. We check that $g$ belongs to $W_{1}$, in particular $g^{\prime \prime} \in L^{\infty}(\mathbb{R})$ in the sense of distribution. Remark as well that $g$ is sublinear.

As for the initial condition to be controlled, we consider simply $u_{0}(x)=\beta \sin (\pi x)$ parametrized by $\beta>0$.

The experiments are performed with the Freefem ++ package developed at the Sorbonne university (see [18]), very well-adapted to the space-time formulation we employ. The algorithm is stopped when the value $E\left(y_{k}, f_{k}\right)$ is smaller than $\epsilon=10^{-6}$. We take the value $m=1$ in (3.11), meaning that the optimal steps $\lambda_{k}$ are searched in the interval $[0,1]$.

Tables 1-3 collect some norms for the sequence $\left(y_{k}, f_{k}\right)_{k \in \mathbb{N}}$ defined by the algorithm (3.11), initialized with the linear controlled solution, for $\beta=10, \beta=10^{2}$ and $\beta=10^{3}$ respectively. We use a structured mesh composed of 20000 triangles, 10201 vertices and for which $h \approx 1.11 \times 10^{-2}$. For $\beta=10$, we observe the convergence after 4 iterations. The optimal steps $\lambda_{k}$ are very close to one since $\max _{k}\left|\lambda_{k}-1\right|<0.05$; consequently, the algorithm (3.11) provides similar results than the Newton algorithm (for which $\lambda_{k}=1$ for all $k$ ). For $\beta=10^{2}$, the convergence remains fast and is reached after 8 iterates. We can observe that some optimal steps differ from one since $\max _{k}\left|\lambda_{k}-1\right|>0.4$. Nevertheless, the Newton algorithm still converges after 17 iterations. More interestingly, the value $\beta=10^{3}$ illustrates the features and robustness of the algorithm: the convergence is achieved after 19 iterations. Far away from a zero of $E$, the variations of the error functional $k \rightarrow E\left(y_{k}, f_{k}\right)$ are first quite slow, then increase to become very fast after 16 iterations, when $\lambda_{k}$ is close to one. In contrast, for $\beta=10^{3}$, the Newton algorithm still initialized with the linear solution diverges (see Tab. 4). As discussed in [22], in that case, a continuation method with respect to the parameter $\beta$ may be combined with the Newton algorithm.

On the contrary, we mention that with these data, the sequences obtained from the algorithm (1.8) based on the linearization introduced in [16], remain bounded but do not converge, including for the value $\beta=10$. The convergence is observed notably with a larger size of the domain $\omega$, for instance $\omega=(0.2,0.8)$ (see [13], Sect. 4.2). 
TABLE 3. Results for the algorithm (3.11); $\beta=10^{3}$.

\begin{tabular}{ccccccc}
\hline \multirow{2}{*}{ iteration $k$} & $\frac{\left\|y_{k}-y_{k-1}\right\|_{L^{2}\left(Q_{T}\right)}}{\left\|y_{k-1}\right\|_{L^{2}\left(Q_{T}\right)}}$ & $\frac{\left\|f_{k}-f_{k-1}\right\|_{L^{2}\left(q_{T}\right)}}{\left\|f_{k-1}\right\|_{L^{2}\left(q_{T}\right)}}$ & $\left\|y_{k}\right\|_{L^{2}\left(Q_{T}\right)}$ & $\left\|f_{k}\right\|_{L^{2}\left(q_{T}\right)}$ & $\sqrt{2 E\left(y_{k}, f_{k}\right)}$ & $\lambda_{k}$ \\
\hline 0 & - & - & 452.80 & 439.18 & $9.809 \times 10^{-1}$ & 0.4215 \\
1 & $8.21 \times 10^{-1}$ & $6.00 \times 10^{-1}$ & 320.12 & 330.15 & $8.536 \times 10^{-1}$ & 0.3919 \\
2 & $6.19 \times 10^{-1}$ & $3.29 \times 10^{-2}$ & 324.02 & 334.12 & $8.012 \times 10^{-1}$ & 0.1566 \\
3 & $4.18 \times 10^{-1}$ & $1.37 \times 10^{-2}$ & 325.65 & 338.21 & $7.953 \times 10^{-1}$ & 0.1767 \\
4 & $3.11 \times 10^{-2}$ & $1.34 \times 10^{-2}$ & 326.11 & 340.12 & $7.851 \times 10^{-1}$ & 0.0937 \\
5 & $2.98 \times 10^{-2}$ & $5.85 \times 10^{-3}$ & 326.35 & 342.24 & $7.688 \times 10^{-2}$ & 0.0491 \\
6 & $3.37 \times 10^{-2}$ & $7.00 \times 10^{-3}$ & 326.91 & 344.65 & $7.417 \times 10^{-2}$ & 0.1296 \\
7 & $4.17 \times 10^{-2}$ & $9.69 \times 10^{-3}$ & 327.23 & 346.12 & $6.864 \times 10^{-2}$ & 0.1077 \\
8 & $2.89 \times 10^{-2}$ & $8.09 \times 10^{-3}$ & 327.42 & 347.19 & $6.465 \times 10^{-2}$ & 0.0859 \\
9 & $1.09 \times 10^{-2}$ & $6.40 \times 10^{-3}$ & 327.49 & 347.29 & $6.182 \times 10^{-2}$ & 0.0968 \\
10 & $1.02 \times 10^{-2}$ & $6.72 \times 10^{-3}$ & 327.92 & 347.38 & $5.805 \times 10^{-2}$ & 0.1184 \\
11 & $6.32 \times 10^{-3}$ & $6.91 \times 10^{-3}$ & 328.13 & 347.41 & $5.371 \times 10^{-2}$ & 0.1730 \\
12 & $5.53 \times 10^{-3}$ & $7.41 \times 10^{-3}$ & 328.16 & 347.43 & $4.825 \times 10^{-2}$ & 0.2579 \\
13 & $4.32 \times 10^{-3}$ & $8.22 \times 10^{-3}$ & 328.19 & 347.45 & $4.083 \times 10^{-2}$ & 0.3817 \\
14 & $2.13 \times 10^{-3}$ & $8.14 \times 10^{-3}$ & 328.21 & 347.48 & $3.164 \times 10^{-2}$ & 0.4946 \\
15 & $3.57 \times 10^{-3}$ & $7.34 \times 10^{-3}$ & 328.22 & 347.50 & $2.207 \times 10^{-2}$ & 0.8294 \\
16 & $1.01 \times 10^{-3}$ & $6.68 \times 10^{-3}$ & 328.25 & 347.51 & $1.174 \times 10^{-2}$ & 0.9845 \\
17 & $5.68 \times 10^{-4}$ & $3.84 \times 10^{-4}$ & 328.26 & 347.51 & $2.191 \times 10^{-3}$ & 0.9999 \\
18 & $2.14 \times 10^{-4}$ & $5.85 \times 10^{-5}$ & 328.26 & 347.52 & $4.674 \times 10^{-5}$ & 1. \\
19 & $3.21 \times 10^{-6}$ & $1.57 \times 10^{-7}$ & 328.27 & 347.52 & $5.843 \times 10^{-7}$ & - \\
\hline
\end{tabular}

TABLE 4. Results for the algorithm (3.11) with $\lambda_{k}=1$ for all $k ; \beta=10^{3}$.

\begin{tabular}{cccccc}
\hline \multirow{2}{*}{ iterate $k$} & $\left\|y_{k}-y_{k-1}\right\|_{L^{2}\left(Q_{T}\right)}$ & $\frac{\left\|f_{k}-f_{k-1}\right\|_{L^{2}\left(q_{T}\right)}}{\left\|y_{k-1}\right\|_{L^{2}\left(Q_{T}\right)}}$ & $\frac{\left\|y_{k}\right\|_{L^{2}\left(Q_{T}\right)}}{\left\|f_{k-1}\right\|_{L^{2}\left(q_{T}\right)}}$ & $\left\|f_{k}\right\|_{L^{2}\left(q_{T}\right)}$ & $\sqrt{2 E\left(y_{k}, f_{k}\right)}$ \\
\hline 0 & - & - & 452.80 & 439.18 & $9.809 \times 10^{-1}$ \\
1 & $9.76 \times 10^{-1}$ & 1.05 & 330.21 & 334.15 & $9.812 \times 10^{-1}$ \\
2 & 1.02 & 1.11 & 344.37 & 336.12 & 1.356 \\
3 & 1.27 & 1.13 & 366.92 & 338.23 & 4.319 \\
4 & 1.18 & 1.25 & 406.06 & 343.12 & 4.799 \\
5 & 1.01 & 1.14 & 481.53 & 405.03 & 13.131 \\
\hline
\end{tabular}

\section{Conclusions}

We have constructed an explicit sequence of functions $\left(f_{k}\right)_{k \in \mathbb{N}}$ converging strongly for the $L^{2}\left(q_{T}\right)$ norm towards a null control for the semilinear heat equation $\partial_{t} y-\Delta y+g(y)=f 1_{\omega}$. The construction of the sequence is based on the minimization of a $L^{2}\left(0, T ; H^{-1}(\Omega)\right)$ least-squares functional. The use of a specific descent direction allows to achieve a global convergence (uniform with respect to the data and to the initial guess) with a superlinear rate related to the regularity of the nonlinear function $g$. Numerical experiments confirm the robustness of the approach. In this analysis, we have assumed in particular that the derivative of $g$ is uniformly bounded in $\mathbb{R}$. This allows to get a uniform bound of the observability constant $C\left(s, \Omega, \omega, T,\left\|g^{\prime}(y)\right\|_{\infty}\right)$ appearing in the estimate (2.5). In order to remove this assumption and be able to consider super-linear function $g$ (as in the seminal work [16] by Fernández-Cara and Zuazua, assuming that $g$ is locally Lipschitz-continuous and the asymptotic behavior (1.6)), the analysis needs to be refined in order to keep as explicit as possible the observability constant $C\left(s, \Omega, \omega, T,\left\|g^{\prime}(y)\right\|_{L^{\infty}}\right)$ in term of the norm $\left\|g^{\prime}(y)\right\|_{L^{\infty}}$ and the parameter $s$ (which appears in the Carleman weights). We refer to [11] for precise estimates of the observability constant. In the one dimensional setting, this 
analysis has been recently made in [21] assuming that the asymptotic behavior $\lim \sup _{|r| \rightarrow \infty} \frac{\left|g^{\prime}(r)\right|}{\ln ^{3 / 2}(1+|r|)} \leq \beta$ for some $\beta>0$ small enough.

We also emphasize that this least-squares approach is very general and may be used to address other PDEs. We also mention the explicit construction of controls for nonlinear wave equation (initially studied in [31] making use of a fixed-point argument) recently performed in [2, 28]. Eventually, as a challenge, it would be interesting to apply the method to approximate controls for the Navier-Stokes system. In this direction, we mention [25] devoted to the Stokes system and $[22,23]$ devoted to the direct problem for the Navier-Stokes system in the incompressible regime.

\section{REFERENCES}

[1] V. Barbu, Exact controllability of the superlinear heat equation. Appl. Math. Optim. 42 (2000) 73-89.

[2] A. Bottois, J. Lemoine and A. Münch, Constructive exact control of semilinear multi-dimensional wave equations. Preprint. arXiv: 2101.06446.

[3] F. Boyer, On the penalised HUM approach and its applications to the numerical approximation of null-controls for parabolic problems, in CANUM 2012, 41e Congrès National d'Analyse Numérique, volume 41 of ESAIM Proc.. EDP Sci., Les Ulis (2013) $15-58$.

[4] F. Boyer and J. Le Rousseau, Carleman estimates for semi-discrete parabolic operators and application to the controllability of semi-linear semi-discrete parabolic equations. Ann. Inst. H. Poincaré Anal. Non Linéaire 31 (2014) 1035-1078.

[5] C. Carthel, R. Glowinski and J.-L. Lions, On exact and approximate boundary controllabilities for the heat equation: a numerical approach. J. Optim. Theory Appl. 82 (1994) 429-484.

[6] T. Cazenave and A. Haraux, An introduction to semilinear evolution equations, Volume 13 of Oxford Lecture Series in Mathematics and its Applications. The Clarendon Press, Oxford University Press, New York (1998). Translated from the 1990 French original by Yvan Martel and revised by the authors.

[7] P.G. Ciarlet, The finite element method for elliptic problems, volume 40 of Classics in Applied Mathematics. Society for Industrial and Applied Mathematics (SIAM), Philadelphia, PA (2002). Reprint of the 1978 original [North-Holland, Amsterdam; MR0520174 (58 \#25001)].

[8] J.-M. Coron, Control and nonlinearity, volume 136 of Mathematical Surveys and Monographs. American Mathematical Society, Providence, RI (2007).

[9] J.-M. Coron and E. Trélat, Global steady-state controllability of one-dimensional semilinear heat equations. SIAM J. Control Optim. 43 (2004) 549-569.

[10] P. Deuflhard, Newton methods for nonlinear problems. Vol. 35 of Springer Series in Computational Mathematics. Springer, Heidelberg (2011). Affine invariance and adaptive algorithms, First softcover printing of the 2006 corrected printing.

[11] T. Duyckaerts, X. Zhang and E. Zuazua, On the optimality of the observability inequalities for parabolic and hyperbolic systems with potentials. Ann. Inst. H. Poincaré Anal. Non Linéaire 25 (2008) 1-41.

[12] E. Fernández-Cara and S. Guerrero, Global Carleman inequalities for parabolic systems and applications to controllability. SIAM J. Control Optim. 45 (2006) 1399-1446.

[13] E. Fernández-Cara and A. Münch, Numerical null controllability of semi-linear 1-D heat equations: fixed point, least squares and Newton methods. Math. Control Relat. Fields 2 (2012) 217-246.

[14] E. Fernández-Cara and A. Münch, Strong convergent approximations of null controls for the 1D heat equation. SeMA J. 61 (2013) 49-78.

[15] E. Fernández-Cara and A. Münch, Numerical exact controllability of the 1D heat equation: duality and Carleman weights. $J$. Optim. Theory Appl. 163 (2014) 253-285.

[16] E. Fernández-Cara and E. Zuazua, Null and approximate controllability for weakly blowing up semilinear heat equations. Ann. Inst. H. Poincaré Anal. Non Linéaire 17 (2000) 583-616.

[17] A.V. Fursikov and O. Yu. Imanuvilov, Controllability of evolution equations, volume 34 of Lecture Notes Series. Seoul National University, Research Institute of Mathematics, Global Analysis Research Center, Seoul (1996).

[18] F. Hecht, New development in Freefem++. J. Numer. Math. 20 (2012) 251-265.

[19] S. Labbé and E. Trélat, Uniform controllability of semidiscrete approximations of parabolic control systems. Systems Control Lett. 55 (2006) 597-609.

[20] K. Le Balc'h, Global null-controllability and nonnegative-controllability of slightly superlinear heat equations. J. Math. Pures Appl. 135 (2020) 103-139.

[21] J. Lemoine and A. Münch, Constructive exact controls for semilinear one-dimensional heat equations. Preprint. arXiv: 2103.09640 .

[22] J. Lemoine and A. Münch, A fully space-time least-squares method for the unsteady Navier-Stokes system. Preprint. arXiv: 1909.05034. In revision in J. of Mathematical Fluid Mechanics.

[23] J. Lemoine and A. Münch, Resolution of the implicit Euler scheme for the Navier-Stokes equation through a least-squares method. Numer. Math. 147 (2021) 349-391.

[24] J. Lemoine, A. Münch and P. Pedregal, Analysis of continuous $H^{-1}$-least-squares approaches for the steady Navier-Stokes system. Appl. Math. Optim. 83 (2021) 461-488. 
[25] A. Münch, A least-squares formulation for the approximation of controls for the Stokes system. Math. Control Signals Syst. 27 (2015) 49-75.

[26] A. Münch and P. Pedregal, Numerical null controllability of the heat equation through a least squares and variational approach. Eur. J. Appl. Math. 25 (2014) 277-306.

[27] A. Münch and D.A. Souza, A mixed formulation for the direct approximation of $L^{2}$-weighted controls for the linear heat equation. Adv. Comput. Math. 42 (2016) 85-125.

[28] A. Münch and E. Trélat, Constructive exact control of semilinear 1d wave equations by a least-squares approach. Preprint. arXiv: 2011.08462.

[29] A. Münch and E. Zuazua, Numerical approximation of null controls for the heat equation: ill-posedness and remedies. Inverse Probl. 26 (2010) 085018.

[30] P. Saramito, A damped Newton algorithm for computing viscoplastic fluid flows. J. Non-Newton. Fluid Mech. 238 (2016) $6-15$.

[31] E. Zuazua, Exact controllability for semilinear wave equations in one space dimension. Ann. Inst. H. Poincaré Anal. Non Linéaire 10 (1993) 109-129. 